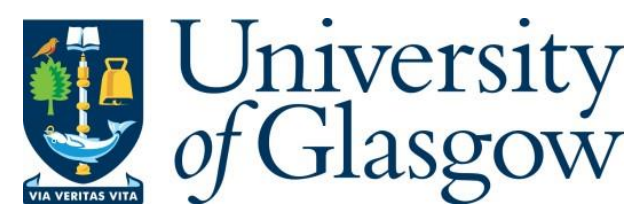

Zhou, J., Tian, D., Sheng, Z., Duan, X., Qu, G., Zhao, D., Cao, D. and Shen, X. (2022) Robust min-max model predictive vehicle platooning with causal disturbance feedback. IEEE Transactions on Intelligent Transportation Systems, (doi:

10.1109/TITS.2022.3146149).

There may be differences between this version and the published version. You are advised to consult the publisher's version if you wish to cite from it.

https://eprints.gla.ac.uk/264897/

Deposited on: 7 February 2022

Enlighten - Research publications by members of the University of Glasgow https://eprints.gla.ac.uk 


\title{
Robust Min-Max Model Predictive Vehicle Platooning With Causal Disturbance Feedback
}

\author{
Jianshan Zhou ${ }^{\circledR}$, Daxin Tian ${ }^{\circledR}$, Senior Member, IEEE, Zhengguo Sheng ${ }^{\circledR}$, Senior Member, IEEE, \\ Xuting Duan ${ }^{\circledR}$, Guixian $\mathrm{Qu}^{\circledR}$, Dezong Zhao $^{\circledR}$, Senior Member, IEEE, \\ Dongpu $\mathrm{CaO}^{\left({ }^{(}\right)}$, Member, IEEE, and Xuemin Shen ${ }^{(}$, Fellow, IEEE
}

\begin{abstract}
Platoon-based vehicular cyber-physical systems have gained increasing attention due to their potentials in improving traffic efficiency, capacity, and saving energy. However, external uncertain disturbances arising from mismatched model errors, sensor noises, communication delays and unknown environments can impose a great challenge on the constrained control of vehicle platooning. In this paper, we propose a closed-loop min-max model predictive control (MPC) with causal disturbance feedback for vehicle platooning. Specifically, we first develop a compact form of a centralized vehicle platooning model subject to external disturbances, which also incorporates the lower-level vehicle dynamics. We then formulate the uncertain optimal control of the vehicle platoon as a worst-case constrained optimization problem and derive its robust counterpart by semidefinite relaxation. Thus, we design a causal disturbance feedback structure with the robust counterpart, which leads to a closed-loop min-max MPC platoon control solution. Even though the min-max MPC follows a centralized paradigm, its robust counterpart can keep the convexity and enable the efficient and practical implementation of current convex optimization techniques. We also derive a linear matrix inequality (LMI)
\end{abstract}

Manuscript received September 15, 2021; revised December 14, 2021; accepted January 19, 2022. This work was supported in part by the National Postdoctoral Program for Innovative Talents under Grant BX2021027, in part by the China Postdoctoral Science Foundation under Grant 2020M680299, in part by the Opening Project of Ministry of Transport Key Laboratory of Technology on Intelligent Transportation Systems under Grant F20211746, in part by the National Natural Science Foundation of China under Grant U20A20155 and Grant 61822101, in part by the Beijing Municipal Natural Science Foundation under Grant L191001, and in part by the Newton Advanced Fellowship under Grant 62061130221. The Associate Editor for this article was I. Papamichail. (Corresponding author: Daxin Tian.)

Jianshan Zhou, Daxin Tian, and Xuting Duan are with the Beijing Advanced Innovation Center for Big Data and Brain Computing, Beijing Key Laboratory for Cooperative Vehicle Infrastructure Systems and Safety Control, School of Transportation Science and Engineering, Beihang University, Beijing 100191, China (e-mail: jianshanzhou@foxmail.com; dtian@buaa.edu.cn; duanxuting@buaa.edu.cn).

Zhengguo Sheng is with the Department of Engineering and Design, University of Sussex, Richmond, Brighton BN1 9RH, U.K. (e-mail: z.sheng@sussex.ac.uk).

Guixian Qu is with the Aero-Engine System Collaborative Design Center, Research Institute of Aero-Engine, Beihang University, Beijing 100191, China (e-mail: guixianqu@foxmail.com).

Dezong Zhao is with the James Watt School of Engineering, University of Glasgow, Glasgow G12 8QQ, U.K. (e-mail: dezong.zhao@glasgow.ac.uk).

Dongpu Cao is with the Department of Mechanical and Mechatronics Engineering, University of Waterloo, Waterloo, ON N2L 3G1, Canada (e-mail: dongpu.cao@uwaterloo.ca).

Xuemin Shen is with the Electrical and Computer Engineering Department, University of Waterloo, Waterloo, ON N2L 3G1, Canada (e-mail: sshen@uwaterloo.ca). condition for guaranteeing the recursive feasibility and inputto-state practical stability (ISpS) of the platoon system. Finally, simulation results are provided to verify the effectiveness and advantage of the proposed MPC in terms of constraint satisfaction, platoon stability and robustness against different external disturbances.

Index Terms-Connected and automated vehicles (CAVs), vehicle platooning, model predictive control (MPC), robust optimization, closed-loop control.

\section{INTRODUCTION}

$\mathbf{R}$ ECENT advances in vehicular networking, communication, and computing technologies have facilitated the practical deployment of connected vehicles [1]-[3]. By joining these advances with modern control technologies, the vehicular system is promising to shift from the individual driving automation to a novel paradigm, i.e., a platoon-based vehicular cyber-physical system (VCPS) [4]-[6]. It is envisioned that the VCPS can improve road traffic safety and efficiency and also bring favorable and environmentally-friendly driving experience. Specifically, the vehicle platooning control of connected and autonomous vehicles (CAVs) has attracted increasing attention in recent years [7]-[10]. In general, the goal of vehicle platooning control is to maintain a desired space headway or a desired time headway between any two successive platooning vehicles meanwhile stabilizing the whole platoon moving at a desired velocity with guaranteed safety. Over the past several decades, considerable research efforts from both academia and industry have been dedicated to the design and analysis of adaptive cruise control (ACC) and cooperative adaptive cruise control (CACC) solutions based on classical linear control theory [11]-[21]. In control theory, the platoon control problem can also be regarded as the consensus control problem of a networked multi-agent system, thus many researchers have been engaged in developing novel consensus-based control approaches for vehicle platooning [10], [22]-[24].

Although vehicle platoon control has been extensively investigated using classical control theory, there exist some challenges to be addressed for the design of a platoon controller, such as the uncertain disturbances resulting from mismatched model errors, sensor measurement errors, wireless communication delays. These challenges can significantly 
affect the control stability and robustness. More importantly, the uncertain disturbances may incur a failure of a platoon controller in satisfying the safety constraint and thus lead to chain collisions. At this point, it is of paramount importance to develop novel approaches that can not only stabilize the vehicle platoon with a desired inter-vehicle spacing and a desired velocity, but also satisfy physical constraints in the presence of external uncertain disturbances.

\section{A. Literature Review}

Early research efforts on vehicle platoon control have been based on the optimal linear-feedback control theory [25], [26]. Besides, many technical efforts have also been dedicated to prototyping and field-testing a vehicle platoon control system, among which the PATH program proposed in $1980 \mathrm{~s}$ is one of the famous and early practices [27]. Since then, extensive studies on this topic have been conducted and considerable linear state feedback control approaches for enabling ACC/CACC or platooning vehicle consensus have been developed in the literature [11]-[24]. For example, [11] has combined a Takagi-Sugeno fuzzy model with a carfollowing controller, in which the fuzzy logic model is used as a predictor for estimating the state sequence of the preceding vehicle. In [12], the impacts of various communication factors such as the actuation lag, vehicular communication period and delay on the CACC of a high-density vehicle platoon have been investigated. In [13], a lane-change strategy is integrated with a ACC system via a model predictive control (MPC)-based coordination algorithm to enable lane changing. In [14], the authors take into account the characteristics of wireless communication links, such as the sampling delays, and present a network-aware CACC approach. In other works [15]-[24], researchers have designed a variety of ACC/CACC control solutions by combining different mechanisms, such as multi-modeling [15], driving style recognition [16], direct yaw moment control [17], acceleration/control feedforward [18], linear quadratic regulator (LQR) [19], and information-delay compensation mechanisms [20]-[24]. Additionally, some researchers incorporate parametric uncertainties into the vehicle dynamics and aim to design robust linear feedback controllers for vehicle platooning by leveraging the classical Kharitonov theorem and the Hurwitz criterion [28]. From the above literature [11]-[24], [28], linear feedback or feedforward-based ACC/CACC approaches have an appealing advantage that they have simple formulations for practical realization and can simplify the analysis of platoon control feasibility and stability. However, these control approaches cannot directly incorporate a platoon control objective and physical constraints such as velocity/acceleration bounds and chain collision-avoidance constraints.

Some advanced lane keeping (LK) and ACC control methods have also been proposed by combining sum-of-squares (SOS) optimization and control barrier functions (CBFs). As in [29], the authors construct the CBFs via the SOS optimization and use the CBFs to obtain the control solutions. The advantage of such a method is that the designed control can guarantee the forward invariance of a set encoding the vehicle safety specifications. However, [29] treats the desired yaw rate as a kind of external disturbances, while the effect of random uncertain disturbances on the longitudinal multi-vehicle platooning dynamics remains unexplored. In [30], some ACC controllers have been designed by using nonlinear functions of inter-vehicle distance and velocity. A simple second-order linear model is adopted as the plant model of [30], which has not allowed for external uncertainties. Since the double-integral model is used for describing each vehicle node, the lower-level dynamics, such as the acceleration variation, cannot be fully considered as well. In [31], the authors propose a distributed platoon control protocol based on state feedback that does not require the prior knowledge of model nonlinearities and disturbances. However, some realistic motion constraints, e.g., the velocity and acceleration constraints to guarantee the mobility smoothness, cannot be taken into account in the controller of [31]. The selection of their controller gains, which affects the control convergence rate, requires empirical tuning techniques. Reference [32] has proposed an efficient delay-based spacing policy for vehicle platoon in the presence of external disturbances. Even though chain collisions can be well avoided in the spacing policy of [32], some real physical constraints on the lower-level dynamics, such as the bounds on the velocity, acceleration and acceleration variation, cannot be incorporated to strictly guarantee dynamics specifications. Besides, the gains of the feedback control in [32] require fine tuning to meet the design-specified objectives (e.g., the fuel-efficiency maximization or the control cost minimization) in actual application scenarios.

Besides the classical linear control-theoretical approaches, various sliding mode control approaches have also been applied to vehicle platooning in the presence of uncertain external disturbances [9], [33]-[38]. In [33], the authors propose a distributed sliding mode control framework to allow for diverse communication topologies in vehicle platoons. Reference [34] designs a robust nonlinear observer that can be integrated with a sliding mode controller to deal with the system uncertainties. Differently, [35] handles communication-topological uncertainties as a set of parametric uncertainties, for which the authors propose a distributed sliding model control approach. In [9], the authors adopt a sliding mode controller for coordinating the vehicles approaching an intersection. In some others [36]-[38], different integral sliding mode controllers have been designed based on the disturbance observation technique to achieve a constant space headway or a constant time headway between platooning vehicles. It is remarked from the existing studies [9], [33]-[38] that combining the sliding mode control methodology with a disturbance observer technique is promising to construct a robust platoon controller, since such a controller can handle both the system nonlinearity and bounded disturbances. Nevertheless, classical sliding mode control may experience the so-called control chattering problem, and they cannot allow for specific objective functions and physical constraints in the controller design.

Another representative methodology for the design of a robust control system is based on the well-developed H-infinity control theory, i.e., the so-called $\mathcal{H}_{\infty}$ control [39]. The 
underlying idea of $\mathcal{H}_{\infty}$ control is to seek an appropriate feedback control law that can minimize the $\mathcal{H}_{2}$ norm or the $\mathcal{H}_{\infty}$ norm of a targeted closed-loop system's transfer matrix mapping external disturbances to relevant outputs [40]. At this point, the optimization technique is allowed to come into play in solving the formulation of the $\mathcal{H}_{\infty}$ control. Thus, this has attracted much attention in the application domain of vehicle platooning [41]-[44]. In [41], multiple $\mathcal{H}_{\infty}$ acceleration-tracking controllers have been designed according to multiple uncertain vehicle dynamics models, and the authors further propose a multiple-model switching-based platoon control solution. In [42], the authors present a distributed $\mathcal{H}_{\infty}$ control approach for a vehicle platoon in the presence of multiplicative uncertainties. Reference [43] considers the mixed traffic scenario in which a platoon consists of both CAVs and human-driven vehicles (HDVs). For the mixed platoon, a car-following strategy is developed based on $\mathcal{H}_{\infty}$ control theory [43]. In [44], the distributed $\mathcal{H}_{\infty}$ controller synthesis approach is also adopted and combined with the matrix spectral decomposition technique to deal with the undirected topologies of a vehicle platoon. It has been shown from the current works [41]-[44] above and the therein references that the $\mathcal{H}_{\infty}$ control theory indeed provides a powerful and robust tool to deal with the system disturbances/uncertainties and also achieve the worst-case performance. However, the synthesis of the $\mathcal{H}_{\infty}$ controller requires the formulation of the transfer function or transfer matrix of the platoon system. This also relies on the matrix decomposition, which may lead to high computational complexity. Besides, in the synthesis procedure, multiple physical constraints on different vehicles' velocities, accelerations or control inputs cannot be incorporated in the control law.

To directly and explicitly incorporate the control objective and constraints of a plant in its control law design, a promising way is to join constrained optimization theory with modern control theory [45]. This fundamental idea has spawned one of the most significant directions in the control domain, i.e., optimal control. Optimal control can be further divided into two categories, model predictive control and rolling horizon control, according to the time horizon for control implementation. In fact, many researchers have leveraged model predictive control (MPC) technique to develop platoon-oriented ACC and CACC controllers like the aforementioned works [11], [13], [16], [17]. In [46], the authors propose a distributed MPC platoon control approach by considering unidirectional topologies. In [47], a MPC model with only one ahead-prediction step is presented and a distributed dual-primal constrained optimization algorithm is applied to solve the designed MPC with the constraint satisfaction. In [48], [49], the authors propose a rolling horizon control framework and a numerical algorithm based on the Pontryagin's principle for the design of non-linear model predictive ACC controllers for both homogeneous and heterogeneous platoons. These works [11], [13], [16], [17], [46]-[49] show that conventional MPC-based approaches enable the transformation from well-defined design specifications to a control objective with a set of constraints. However, their MPC formulations have not taken into consideration exogenous disturbances and thus cannot guarantee the system robustness, in particular, the worst-case control performance and constraint satisfaction.

Additionally, to develop robust MPC solutions, some other studies integrate the point parameter estimation and the tube construction-based approach to deal with parametric uncertainties and additive disturbances [50], [51]. For example, [52] presents a tube MPC model for mixed vehicle platooning, where a tube, formed by a series of sets that contain the prediction uncertainties of HDVs with a large probability, is dynamically constructed by using a feedforward controller and used to restrict the CAVs' motion trajectories. Different from tube-based solutions, some works like [53], [54] have integrated parametric estimation and compensation techniques with stochastic optimization theory to formulate MPC models. In [53], the authors have formulated the MPC problem as a linearly constrained linear quadratic Gaussian (LQG) problem and thus relied on a Kalman filter to address the control system's and sensors' disturbances that are normally distributed. In [54], the authors also combine a conditional linear Gaussian estimator with a MPC model to handle the normallydistributed disturbances, in which the Gaussian model is used for the probability distribution estimation of the velocity prediction of a preceding vehicle. From the above works [53], [54], it is seen that the integration of parametric estimation (e.g., Kalman filter) and stochastic optimization with MPC can improve the robustness of vehicle platooning. But these robust MPC solutions are limited by the underlying assumption on the normally-distributed disturbances. Additionally, the tube-based MPC approaches usually result in conservative design that cannot sufficiently exploit the potential of feedback control.

To relax the probability distribution-related assumption for uncertain disturbances, another type of robust MPC paradigm, so-called min-max MPC, is developed by leveraging robust optimization theory. The fundamental goal of min-max MPC is to design a constraint-satisfied control law that can optimize the worst-case performance criterion. In particular, robust invariant sets, semidefinite programming relaxation, linear matrix inequality (LMI) and some other modeling techniques in robust optimization are used to transform uncertain objective functions and constraints to their robust counterpart approximations [55]-[58]. Moreover, min-max MPC has also been combined with time-discrete self-triggered mechanisms to facilitate their implementation with low-computation and low-communication burden [59], [60]. Thus, some works like [61] have applied the min-max MPC approach to the design of a robust platoon controller in the presence of actuator lags. In our previous work [62], a robust min-max MPC controller has been proposed to deal with the communication delay between any two successive vehicles in a platoon. However, [62] only considers a second-order model (i.e., a double-integral model) to characterize the vehicle behavior, and thus does not capture the lower-layer vehicle dynamics. Besides, the min-max MPC models of [61] and [62] have not coped with the exogenous uncertain disturbances. To address the issue of control conservatism in open-loop min-max MPC approaches, a feedback technique is usually combined with the min-max optimization. However, the introduction of state feedback may lead to the nonlinearity of a closed-loop 
min-max optimization formulation. It requires much computation power and efficient algorithms to solve nonlinear MPC problems [55]-[57].

\section{B. Motivation and Contribution}

When compared to other methodologies, MPC approaches are more appealing because of their flexibility to define an optimization objective function and a set of constraints. Nevertheless, it remains a great challenge to design a highly efficient algorithm to deal with the potential complexity in a closed-loop MPC formulation. On the other side, a standard approach to reduce the conservatism in the MPC is to use the feedback technique, e.g., the state feedback. But the conventional state feedback can result in the nonlinearity in the closed-loop MPC, and thus increase the computational complexity. Few studies have been made on the design and implementation of a robust min-max MPC-based vehicle platoon controller that can sufficiently exploit the historical information contained in the past disturbances and capture the causal impact of the disturbances into the future control input.

Towards this end, this paper proposes a closed-loop minmax MPC model to facilitate the robust control of vehicle platooning in the presence of external uncertain but bounded disturbances. In the formulation of the proposed closed-loop min-max MPC, a causal disturbance feedback is introduced to the control design, i.e., using uncertainties to parameterize the control input via a causal structure. It is highlighted that even though the closed-loop min-max MPC needs the centralized computation implementation, its integration with the causal disturbance feedback can keep the mathematical convexity and thus enable legacy convex optimization techniques to come into play in practice. Specifically, the main contributions of our paper are summarized as follows:

i) A closed-loop min-max MPC system model is proposed based on a causal disturbance feedback for robust vehicle platooning with consideration of external uncertainties. In this model, the control input of each platooning vehicle is parameterized with the disturbances in a causal structure, such that the impact of the historical disturbances can be captured in the design of the future control sequence. The proposed model can not only reduce the control conservatism but also facilitate the highly-efficient control implementation by leveraging existing convex optimization techniques.

ii) We also present theoretical analysis on the recursive feasibility and the input-to-state practical stability (ISpS) of the proposed min-max MPC platoon control. We derive a linear matrix inequality (LMI) condition by using the semidefinite relaxation technique and the Schur complement. The platoon control approach under the LMI condition is proved to be recursively feasible and input-to-state practical stable.

iii) We conduct simulations to verify the proposed platoon control method. Additionally, we illustrate the superior performance achieved by the proposed method over other conventional methods, i.e., the conventional MPC and the LQG controllers, in terms of platoon stability and robustness.

The remainder of the paper is organized as follows. In Section II, we model the longitudinal platooning dynam-

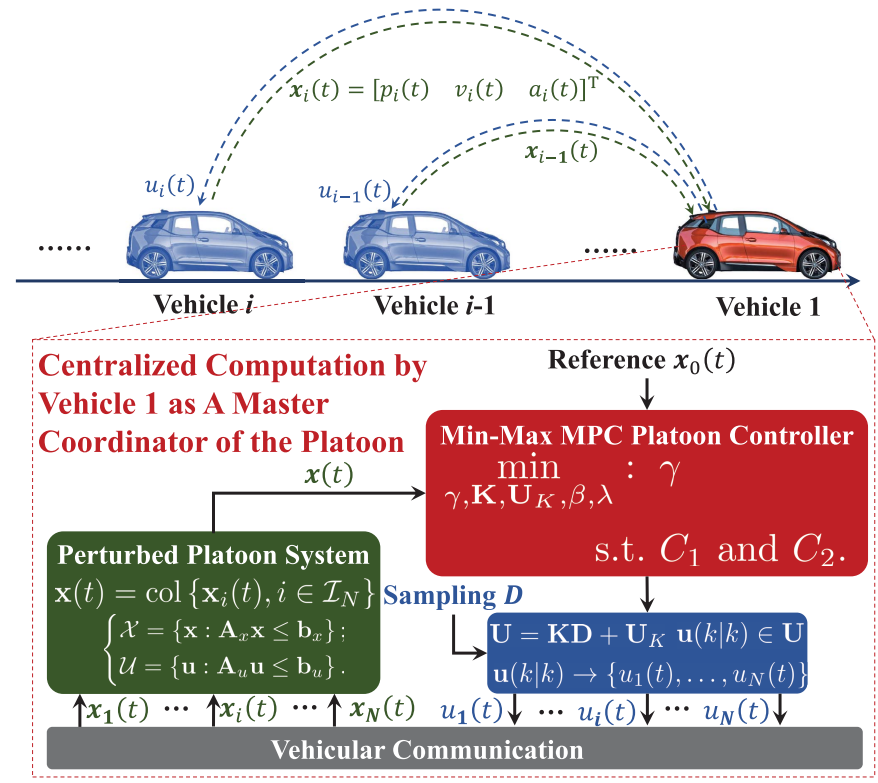

Fig. 1. A system scenario of vehicle platooning and an implementation framework of the proposed control approach.

ics in the presence of external uncertain disturbances. In Section III, a min-max MPC platoon model is formulated. Section IV proposes the closed-loop control design with a causal disturbance feedback and Section V proves its recursive feasibility and ISpS, which is followed by the performance evaluation in Section VI. Finally, Section VII concludes this paper and also remarks our future work.

\section{Longitudinal Platooning Dynamics With UNCERTAIN DISTURBANCES}

We take into account the longitudinal dynamics of vehicles for platooning control design. To be specific, let $p_{i}(t), v_{i}(t)$, and $a_{i}(t)$ denote the longitudinal position, velocity, and acceleration of vehicle $i$ at time $t$, respectively. The body length of vehicle $i$ is defined as $l_{i}$. The desired time headway for ensuring the safe inter-vehicle distance is specified to $\Delta t$ seconds. For simplicity, the set of the platooning vehicles including a virtual leader is denoted by $\mathcal{I}_{N+1}$, i.e., $\mathcal{I}_{N+1}=\{0,1, \ldots, N\}$, where the virtual leading vehicle of the platoon is indexed by 0 . The set of the platooning vehicles excluding the leader is denoted by $\mathcal{I}_{N}$, i.e., $\mathcal{I}_{N}=\mathcal{I}_{N+1}-\{0\}$. As suggested by current literature, a lower-level vehicle dynamics model can be approximated by considering the actuation lag and attenuation of the control, which is modeled as follows [46], [63], [64],

$$
\dot{a}_{i}(t)=-\frac{1}{\tau} a_{i}(t)+\frac{\kappa}{\tau} u_{i}(t), \quad i \in \mathcal{I}_{N},
$$

where $u_{i}(t)$ denotes the input control signal at time $t, \tau$ is the time lag for actuating a vehicle to realize its required acceleration, and $\kappa$ is the ratio of the actually achieved acceleration over the required acceleration. It is noted that the model (1) allows for incorporating the effects arising from aerodynamic drag, road friction conditions, gear transmission loss, etc., into the vehicle dynamics modeling. Thus, it extends the 
basic second-order model (i.e., the double-integrator model) considered in many existing works, e.g., [23], [47]-[49].

A typical system scenario of vehicle platooning is shown in Figure 1, where the first vehicle of the platoon can exchange information with each other via vehicular wireless communications. The desired space between any two successive vehicles $i-1$ and $i$ in the equilibrium state can be expressed as $v_{i}(t) \Delta t+l_{i-1}$. Thus, the longitudinal position and velocity errors between $i-1$ and $i$ are formulated by

$$
\left\{\begin{array}{l}
e_{1, i}(t)=p_{i-1}(t)-p_{i}(t)-\left(v_{i}(t) \Delta t+l_{i-1}\right) ; \\
e_{2, i}(t)=v_{i-1}(t)-v_{i}(t)
\end{array}\right.
$$

for all $i \in \mathcal{I}_{N}$. It is remarked that, in a practical design, the constant term $l_{i-1}$ can be flexibly configured to be larger than the actual body length of vehicle $i-1$, i.e., set to the vehicle's size plus a certain space margin, so as to avoid rear-end collisions. Combining (1) and (2), we arrive at the following three-order dynamics model

$$
\left\{\begin{array}{l}
\dot{e}_{1, i}(t)=e_{2, i}(t)-a_{i}(t) \Delta t \\
\dot{e}_{2, i}(t)=a_{i-1}(t)-a_{i}(t) \\
\dot{a}_{i}(t)=-\frac{1}{\tau} a_{i}(t)+\frac{\kappa}{\tau} u_{i}(t)
\end{array}\right.
$$

for all $i \in \mathcal{I}_{N}$. In addition, the model (3) can be further re-arranged as another state-space form by defining a state variable $\boldsymbol{x}_{i}(t)=\left[e_{1, i}(t), e_{2, i}(t), a_{i}(t)\right]^{\mathrm{T}}$

$$
\dot{\boldsymbol{x}}_{i}(t)=\boldsymbol{A} \boldsymbol{x}_{i}(t)+\boldsymbol{B} u_{i}(t)+\boldsymbol{C} \boldsymbol{x}_{i-1}(t),
$$

where the coefficient matrices $\boldsymbol{A}, \boldsymbol{B}$, and $\boldsymbol{C}$ are given by

$$
\boldsymbol{A}=\left[\begin{array}{ccc}
0 & 1 & -\Delta t \\
0 & 0 & -1 \\
0 & 0 & -\frac{1}{\tau}
\end{array}\right], \quad \boldsymbol{B}=\left[\begin{array}{c}
0 \\
0 \\
\frac{\kappa}{\tau}
\end{array}\right], \quad \boldsymbol{C}=\left[\begin{array}{lll}
0 & 0 & 0 \\
0 & 0 & 1 \\
0 & 0 & 0
\end{array}\right] .
$$

To proceed, we define the state of the entire platoon system by $\boldsymbol{x}(t)=\operatorname{col}\left\{\boldsymbol{x}_{i}(t), i \in \mathcal{I}_{N}\right\}$, and the global control by $\boldsymbol{u}(t)=$ $\operatorname{col}\left\{u_{i}(t), i \in \mathcal{I}_{N}\right\}$. Let state matrices $\boldsymbol{Q}_{A} \in \mathbb{R}^{3 N \times 3 N}, \boldsymbol{Q}_{B} \in$ $\mathbb{R}^{3 N \times N}$, and $\boldsymbol{Q}_{C} \in \mathbb{R}^{3 N \times 3}$ be

$$
\begin{aligned}
\boldsymbol{Q}_{A} & =\left[\begin{array}{cccccc}
\boldsymbol{A} & \mathbf{0} & \mathbf{0} & \cdots & \mathbf{0} & \mathbf{0} \\
\boldsymbol{C} & \boldsymbol{A} & \mathbf{0} & \cdots & \mathbf{0} & \mathbf{0} \\
\vdots & \vdots & \vdots & \vdots & \vdots & \vdots \\
\mathbf{0} & \mathbf{0} & \mathbf{0} & \cdots & \boldsymbol{C} & \boldsymbol{A}
\end{array}\right], \\
\boldsymbol{Q}_{B} & =\operatorname{diag}\left\{\boldsymbol{B}, i \in \mathcal{I}_{N}\right\}, \\
\boldsymbol{Q}_{C} & =\operatorname{col}\left\{\boldsymbol{C}, \mathbf{0}_{3 \times 3}, \ldots, \mathbf{0}_{3 \times 3}\right\},
\end{aligned}
$$

respectively. The entire platooning system is then represented by the following dynamics

$$
\dot{\boldsymbol{x}}(t)=\boldsymbol{Q}_{A} \boldsymbol{x}(t)+\boldsymbol{Q}_{B} \boldsymbol{u}(t)+\boldsymbol{Q}_{C} \boldsymbol{x}_{0}(t) .
$$

Without loss of generality, the dynamics of the leading vehicle in the platoon can be treated as the reference for all the other following vehicles. Its state $\boldsymbol{x}_{0}(t)$ can be designed in advance. For simplicity, we consider that the leading vehicle is moving at a desired velocity $v_{0}$. For example, $v_{0}$ can be designed as an eco fuel-efficient velocity of the platoon. Therefore, we can set $\boldsymbol{x}_{0}(t)=\mathbf{0}_{3 \times 1}$ at the equilibrium state.
It is recognized that (7) is a time-continuous model. By selecting a proper sample time slot $\tau_{s}$, it can be discretized into a time-discrete form as follows

$$
\boldsymbol{x}(k+1)=\boldsymbol{M}_{A} \boldsymbol{x}(k)+\boldsymbol{M}_{B} \boldsymbol{u}(k),
$$

where $k$ is the index of a time slot. $\boldsymbol{M}_{A}$ and $\boldsymbol{M}_{B}$ are obtained by applying the integral approximation method to $\boldsymbol{Q}_{A}$ and $\boldsymbol{Q}_{B}$, i.e.,

$$
\begin{aligned}
& \boldsymbol{M}_{A}=\exp \left(\boldsymbol{Q}_{A} \tau_{s}\right), \\
& \boldsymbol{M}_{B}=\int_{0}^{\tau_{s}} \exp \left(\boldsymbol{Q}_{A} s\right) d s \times \boldsymbol{Q}_{B} .
\end{aligned}
$$

In fact, the time-discrete linear model (8) has been widely adopted in the current literature. However, there inevitably exist modeling errors due to the mismatched linearized and discretized dynamics. Additionally, (8) cannot capture the impacts of various uncertainties and disturbances in actual environments. Hence, a more realistic model should incorporate the comprehensive effect arising from the mismatched model uncertainties, sensor-based measurement errors, and unknown external disturbances, if any. Let $\boldsymbol{d}(k) \in \mathbb{R}^{3 N \times 1}$ be a lumped uncertainty of the platooning dynamics. By incorporating $\boldsymbol{d}(k)$ into (8), we further derive

$$
\boldsymbol{x}(k+1)=\boldsymbol{M}_{A} \boldsymbol{x}(k)+\boldsymbol{M}_{B} \boldsymbol{u}(k)+\boldsymbol{M}_{D} \boldsymbol{d}(k),
$$

where $\boldsymbol{M}_{D}$ is a known matrix with a compatible dimension. Moreover, while the disturbance $\boldsymbol{d}(t)$ is uncertain, it is usually bounded in practice. We denote the uncertainty set, i.e., the set of possible $\boldsymbol{d}(k)$, by $\mathcal{D}$, and assume that the external unknown disturbances are box-constrained, i.e., $\mathcal{D}=\left\{\boldsymbol{d}:\|\boldsymbol{d}\|_{\infty} \leq 1\right\}$.

To ensure the mobility safety, we also need to take into consideration actual physical constraints on both the state and control variables in (10). Let $\Delta p^{-}, \Delta p^{+}$and $\Delta v^{-}, \Delta v^{+}$be the lower and upper bounds on the inter-vehicle longitudinal position and velocity differences, respectively, for guaranteeing rear-end collision avoidance, i.e., $\Delta p^{-} \leq e_{1, i}(k) \leq \Delta p^{+}$ and $\Delta v^{-} \leq e_{2, i}(k) \leq \Delta v^{+}$for all $i \in \mathcal{I}_{N}$ and all $k . a^{-}$and $a^{+}$ denote the minimum and the maximum allowed accelerations of any vehicle $i$, respectively, i.e., $a^{-} \leq a_{i}(k) \leq a^{+}$for all $i \in \mathcal{I}_{N}$ and all $k$. We can construct the lower and upper bounds on the state of vehicle $i$ as $\boldsymbol{x}_{i}^{-} \leq \boldsymbol{x}_{i}(k) \leq \boldsymbol{x}_{i}^{+}$where $\boldsymbol{x}_{i}^{-}=\left[\Delta p^{-}, \Delta v^{-}, a^{-}\right]^{\mathrm{T}}$ and $\boldsymbol{x}_{i}^{+}=\left[\Delta p^{+}, \Delta v^{+}, a^{+}\right]^{\mathrm{T}}$. Then, the bounds on the global state variable $\boldsymbol{x}(k)$ can be represented as $\boldsymbol{x}^{-}=\operatorname{col}\left\{\boldsymbol{x}_{i}^{-}, i \in \mathcal{I}_{N}\right\}$ and $\boldsymbol{x}^{+}=\operatorname{col}\left\{\boldsymbol{x}_{i}^{+}, i \in \mathcal{I}_{N}\right\}$, respectively. Thus, we have

$$
\boldsymbol{x}^{-} \leq \boldsymbol{x}(k) \leq \boldsymbol{x}^{+}
$$

for all $k$. In addition, to avoid such a situation in which the velocity of a certain vehicle $i$ is negative, we also bound the velocity of each vehicle $i$ as $v_{\min } \leq v_{i}(k) \leq v_{\max }$ for all $i$ and $k$, where $v_{\min }$ and $v_{\max }$ are the minimum and maximum velocities for each vehicle, respectively. Now, recalling (2), we can have $\sum_{l=1}^{i}\left(v_{l-1}(k)-v_{l}(k)\right)=\sum_{l=1}^{i} e_{2, l}(k)=v_{0}(k)-$ $v_{i}(k)$ and thus establish the following state mapping

$$
v_{i}(k)=v_{0}(k)-\sum_{l=1}^{N} z_{i, l} \boldsymbol{x}_{l}(k), \quad i \in \mathcal{I}_{N},
$$


where $z_{i, l}$ is a $1 \times 3$ row vector defined as follows

$$
z_{i, l}= \begin{cases}{[0,1,0],} & 1 \leq l \leq i \\ {[0,0,0],} & i+1 \leq l \leq N .\end{cases}
$$

Let $z_{i}=\left[z_{i, 1}, z_{i, 2}, \ldots, z_{i, N}\right]$. Recalling the definition of the global state variable $\boldsymbol{x}(k),(12)$ can be reshaped as a linear function of $\boldsymbol{x}(k)$

$$
v_{i}(k)=v_{0}(k)-z_{i} x(k), \quad i \in \mathcal{I}_{N} .
$$

Substituting (14) into the velocity bound inequality can yield

$$
v_{0}(k)-v_{\max } \leq z_{i} x(k) \leq v_{0}(k)-v_{\min }, \quad i \in \mathcal{I}_{N} .
$$

Hence, we are able to further represent the linear equality (15) into a more compact form

$$
\boldsymbol{q}_{x}^{-} \leq \boldsymbol{Z}_{x} \boldsymbol{x}(k) \leq \boldsymbol{q}_{x}^{+},
$$

where $\boldsymbol{q}_{x}^{-}=\operatorname{col}\left\{v_{0}(k)-v_{\max }, i \in \mathcal{I}_{N}\right\}$ and $\boldsymbol{q}_{x}^{+}=\operatorname{col}\left\{v_{0}(k)-\right.$ $\left.v_{\min }, i \in \mathcal{I}_{N}\right\} . Z_{x}$ is a $N \times 3 N$ matrix defined by vertically stacking the row vector $z_{i}$, i.e., $\boldsymbol{Z}_{x}=\operatorname{col}\left\{z_{i}, i \in \mathcal{I}_{N}\right\}$. Similarly, the lower and the upper bounds on the controls are also presented by $\boldsymbol{u}_{\min }=\operatorname{col}\left\{u_{\min , i}, i \in \mathcal{I}_{N}\right\}$ and $\boldsymbol{u}_{\max }=$ $\operatorname{col}\left\{u_{\max , i}, i \in \mathcal{I}_{N}\right\}$, respectively. Thus, the bound constraints on the control sequence can be

$$
\boldsymbol{u}_{\min } \leq \boldsymbol{u}(k) \leq \boldsymbol{u}_{\max } .
$$

Now, for the simplicity of notation, let $\mathcal{X}$ and $\mathcal{U}$ be the state and the control constraint sets, i.e., $\boldsymbol{x}(k) \in \mathcal{X}$ and $\boldsymbol{u}(k) \in \mathcal{U}$ for all $k$. Based on (11), (16) and (17), the physical constraints for guaranteeing the platoon safety can be represented by

$$
\left\{\begin{array}{l}
\mathcal{X}=\left\{\boldsymbol{x}: \boldsymbol{A}_{x} \boldsymbol{x} \leq \boldsymbol{b}_{x}\right\}, \\
\mathcal{U}=\left\{\boldsymbol{u}: \boldsymbol{A}_{u} \boldsymbol{u} \leq \boldsymbol{b}_{u}\right\},
\end{array}\right.
$$

where we let $\boldsymbol{A}_{x}=\operatorname{col}\left\{\boldsymbol{I}_{3 N \times 3 N},-\boldsymbol{I}_{3 N \times 3 N}, \boldsymbol{Z}_{x},-\boldsymbol{Z}_{x}\right\}$ and $\boldsymbol{b}_{x}=\operatorname{col}\left\{\boldsymbol{x}^{+},-\boldsymbol{x}^{-}, \boldsymbol{q}_{x}^{+},-\boldsymbol{q}_{x}^{-}\right\}$, respectively. $\boldsymbol{A}_{u}$ and $\boldsymbol{b}_{u}$ are defined as $\boldsymbol{A}_{u}=\operatorname{col}\left\{\boldsymbol{I}_{N \times N},-\boldsymbol{I}_{N \times N}\right\}$ and $\boldsymbol{b}_{u}=$ $\operatorname{col}\left\{\boldsymbol{u}_{\max },-\boldsymbol{u}_{\min }\right\}$, respectively.

\section{Robust Min-MAX MPC FORMULATION FOR Vehicle Platooning}

Basically, the control objective of vehicle platooning can be described based on the system (10) as $\lim _{k \rightarrow \infty}\|\boldsymbol{x}(k)\|=$ 0 under the constraints of $\boldsymbol{x}(k) \in \mathcal{X}, \boldsymbol{u}(k) \in \mathcal{U}$ and $\boldsymbol{d}(k) \in$ $\mathcal{D}$ at all $k$. To achieve this goal, we resort to the min-max optimization, which would like to minimize the worst-case global cost over the constraint sets. Specifically, let $H \in \mathbb{N}_{\geq 1}$ be the prediction horizon for the control implementation of the platoon system. $\boldsymbol{x}(k+j \mid k), \boldsymbol{u}(k+j \mid k)$ and $\boldsymbol{d}(k+j \mid k)$ denote the predicted system state, the predicted control, and the unknown disturbance predicted at $k+j$, respectively, under the condition that the system state information is available at $k$, i.e., $\boldsymbol{x}(k \mid k)$ is known, where $j=1,2, \ldots, H$. The global cost function at $k$ is formulated as

$$
\mathcal{J}(k)=\sum_{j=0}^{H-1} \mathcal{L}(\boldsymbol{x}(k+j \mid k), \boldsymbol{u}(k+j \mid k))+\Phi(\boldsymbol{x}(k+H \mid k)),
$$

where $\mathcal{L}(\boldsymbol{x}(k+j \mid k), \boldsymbol{u}(k+j \mid k))$ and $\Phi(\boldsymbol{x}(k+H \mid k))$ are the stage cost function and the terminal cost function, respectively. These two functions can be usually designed by using a quadratic form. Namely, by specifying three positive definite diagonal matrices with the compatible dimension, $\boldsymbol{w}_{x} \succ 0$, $\boldsymbol{w}_{u} \succ 0$, and $\boldsymbol{V} \succ 0$, we formulate the stage cost and the terminal cost using the quadratic functions as follows

$$
\mathcal{L}(\boldsymbol{x}, \boldsymbol{u})=\boldsymbol{x}^{\mathrm{T}} \boldsymbol{w}_{x} \boldsymbol{x}+\boldsymbol{u}^{\mathrm{T}} \boldsymbol{w}_{u} \boldsymbol{u}, \quad \Phi(\boldsymbol{x})=\boldsymbol{x}^{\mathrm{T}} \boldsymbol{V} \boldsymbol{x} .
$$

For the simplicity of notation, we introduce the compact forms of the predicted system states, controls, and uncertain disturbances over the prediction horizon as $\boldsymbol{X}, \boldsymbol{U}$, and $\boldsymbol{D}$, i.e.,

$$
\left\{\begin{array}{l}
\boldsymbol{X}=\operatorname{col}\{\boldsymbol{x}(k+j \mid k), j=1,2, \ldots, H\} \\
\boldsymbol{U}=\operatorname{col}\{\boldsymbol{u}(k+j \mid k), j=0,1, \ldots, H-1\} \\
\boldsymbol{D}=\operatorname{col}\{\boldsymbol{d}(k+j \mid k), j=0,1, \ldots, H-1\} .
\end{array}\right.
$$

Moreover, we introduce the following block matrices $\boldsymbol{G}_{A} \in$ $\mathbb{R}^{3 H N \times 3 N}, \boldsymbol{G}_{B} \in \mathbb{R}^{3 H N \times H N}$, and $\boldsymbol{G}_{D} \in \mathbb{R}^{3 H N \times 3 H N}$ to reshape the whole system (10) over the finite prediction horizon as

$$
\boldsymbol{X}=\boldsymbol{G}_{A} \boldsymbol{x}(k \mid k)+\boldsymbol{G}_{B} \boldsymbol{U}+\boldsymbol{G}_{D} \boldsymbol{D},
$$

where $\boldsymbol{G}_{A}, \boldsymbol{G}_{B}$, and $\boldsymbol{G}_{D}$ are defined by

$$
\begin{aligned}
\boldsymbol{G}_{A}= & \operatorname{col}\left\{\boldsymbol{M}_{A}^{j}, j=1,2, \ldots, H\right\}, \\
\boldsymbol{G}_{B}= & {\left[\begin{array}{ccccc}
\boldsymbol{M}_{B} & \mathbf{0}_{3 N \times N} & \mathbf{0}_{3 N \times N} & \cdots & \mathbf{0}_{3 N \times N} \\
\boldsymbol{M}_{A} \boldsymbol{M}_{B} & \boldsymbol{M}_{B} & \mathbf{0}_{3 N \times N} & \cdots & \mathbf{0}_{3 N \times N} \\
\boldsymbol{M}_{A}^{2} \boldsymbol{M}_{B} & \boldsymbol{M}_{A} \boldsymbol{M}_{B} & \boldsymbol{M}_{B} & \cdots & \mathbf{0}_{3 N \times N} \\
\vdots & \vdots & \vdots & \vdots & \vdots \\
\boldsymbol{M}_{A}^{H-1} \boldsymbol{M}_{B} & \boldsymbol{M}_{A}^{H-2} \boldsymbol{M}_{B} & \boldsymbol{M}_{A}^{H-3} \boldsymbol{M}_{B} & \cdots & \boldsymbol{M}_{B}
\end{array}\right], } \\
\boldsymbol{G}_{D}= & {\left[\begin{array}{ccccc}
\boldsymbol{M}_{D} & \mathbf{0}_{3 N \times 3 N} & \mathbf{0}_{3 N \times 3 N} & \cdots & \mathbf{0}_{3 N \times 3 N} \\
\boldsymbol{M}_{A} \boldsymbol{M}_{D} & \boldsymbol{M}_{D} & \mathbf{0}_{3 N \times 3 N} & \cdots & \mathbf{0}_{3 N \times 3 N} \\
\boldsymbol{M}_{A}^{2} \boldsymbol{M}_{D} & \boldsymbol{M}_{A} \boldsymbol{M}_{D} & \boldsymbol{M}_{D} & \cdots & \mathbf{0}_{3 N \times 3 N} \\
\vdots & \vdots & \vdots & \vdots & \vdots \\
\boldsymbol{M}_{A}^{H-1} \boldsymbol{M}_{D} & \boldsymbol{M}_{A}^{H-2} \boldsymbol{M}_{D} & \boldsymbol{M}_{A}^{H-3} \boldsymbol{M}_{D} & \cdots & \boldsymbol{M}_{D}
\end{array}\right] . }
\end{aligned}
$$

In addition, we can also obtain the constraint sets of $\boldsymbol{X}, \boldsymbol{U}$, and $\boldsymbol{D}$, respectively, as follows

$$
\begin{aligned}
& \boldsymbol{X} \in \mathbb{X}=\left\{\boldsymbol{X} \mid \begin{array}{l}
\boldsymbol{A}_{X} \boldsymbol{X} \leq \boldsymbol{b}_{X} \\
\boldsymbol{A}_{X}=\operatorname{diag}\left\{\boldsymbol{A}_{x}, j=1,2, \ldots, H\right\} \\
\boldsymbol{b}_{X}=\operatorname{col}\left\{\boldsymbol{b}_{x}, j=1,2, \ldots, H\right\}
\end{array}\right\}, \\
& \boldsymbol{U} \in \mathbb{U}=\left\{\begin{array}{l}
\boldsymbol{U} \mid \begin{array}{l}
\boldsymbol{A}_{U} \boldsymbol{U} \leq \boldsymbol{b}_{U} \\
\boldsymbol{A}_{U}=\operatorname{diag}\left\{\boldsymbol{A}_{u}, j=1,2, \ldots, H\right\} \\
\boldsymbol{b}_{U}=\operatorname{col}\left\{\boldsymbol{b}_{u}, j=1,2, \ldots, H\right\}
\end{array}
\end{array}\right\}, \\
& \boldsymbol{D} \in \mathbb{D}=\prod_{j=1}^{H} \mathcal{D} .
\end{aligned}
$$


Now, by letting

$$
\left\{\begin{array}{l}
\boldsymbol{W}_{X}=\operatorname{diag}\{\underbrace{\boldsymbol{w}_{X}, \ldots, \boldsymbol{w}_{X}}_{H-1}, \boldsymbol{V}\}, \\
\boldsymbol{W}_{U}=\operatorname{diag}\{\underbrace{\boldsymbol{w}_{U}, \ldots, \boldsymbol{w}_{U}}_{H}\},
\end{array}\right.
$$

we can rewrite (19) as

$$
\mathcal{J}(k)=\boldsymbol{x}^{\mathrm{T}}(k \mid k) \boldsymbol{w}_{x} \boldsymbol{x}(k \mid k)+J(\boldsymbol{X}, \boldsymbol{U}),
$$

where $J(\boldsymbol{X}, \boldsymbol{U})$ is given by

$$
J(\boldsymbol{X}, \boldsymbol{U})=\boldsymbol{X}^{\mathrm{T}} \boldsymbol{W}_{X} \boldsymbol{X}+\boldsymbol{U}^{\mathrm{T}} \boldsymbol{W}_{U} \boldsymbol{U} .
$$

Recalling that the initial state $\boldsymbol{x}(k \mid k)$ is known at $k$, $\boldsymbol{x}^{\mathrm{T}}(k \mid k) \boldsymbol{w}_{x} \boldsymbol{x}(k \mid k)$ is a constant in (26). The optimization of the global cost $\mathcal{J}(k)$ is equivalent to optimize $J(\boldsymbol{X}, \boldsymbol{U})$. Thus, following the robust optimization philosophy, we formulate the min-max MPC model that aims to minimize the cost function $J(\boldsymbol{X}, \boldsymbol{U})$ in the worst-case situation since it contains bounded uncertainties

$$
\begin{aligned}
& \min _{\boldsymbol{U}} \max _{\boldsymbol{D}}: \quad J(\boldsymbol{X}, \boldsymbol{U}) \\
& \text { s.t. }\left\{\begin{array}{l}
\boldsymbol{X}=\boldsymbol{G}_{A} \boldsymbol{x}(k \mid k)+\boldsymbol{G}_{B} \boldsymbol{U}+\boldsymbol{G}_{D} \boldsymbol{D}, \\
\boldsymbol{X} \in \mathbb{X}, \quad \forall \boldsymbol{D} \in \mathbb{D}, \\
\boldsymbol{U} \in \mathbb{U}, \quad \forall \boldsymbol{D} \in \mathbb{D} .
\end{array}\right.
\end{aligned}
$$

From (28), it is clear that it is difficult or even impossible to solve the min-max optimization problem exactly. Instead, we further transform (28) into an epigraph formulation by additionally introducing an auxiliary variable $\gamma$ as follows, which can motivate an efficient solution

$$
\begin{array}{ll}
\min _{\boldsymbol{U}, \gamma}: & \gamma \\
\text { s.t. } & \left\{\begin{array}{l}
\boldsymbol{X}=\boldsymbol{G}_{A} \boldsymbol{x}(k \mid k)+\boldsymbol{G}_{B} \boldsymbol{U}+\boldsymbol{G}_{D} \boldsymbol{D}, \\
\boldsymbol{X}^{\mathrm{T}} \boldsymbol{W}_{X} \boldsymbol{X}+\boldsymbol{U}^{\mathrm{T}} \boldsymbol{W}_{U} \boldsymbol{U} \leq \gamma, \forall \boldsymbol{D} \in \mathbb{D}, \\
\boldsymbol{X} \in \mathbb{X}, \quad \forall \boldsymbol{D} \in \mathbb{D}, \\
\boldsymbol{U} \in \mathbb{U}, \quad \forall \boldsymbol{D} \in \mathbb{D} .
\end{array}\right.
\end{array}
$$

From the epigraph formulation (29) above, we can find that the robust control performance can be achieved by ensuring robust satisfaction of the uncertain constraints. That is, we need to perform the constrained minimization over all the admissible uncertain disturbances $\boldsymbol{D} \in \mathbb{D}$. In the following, our main results aim to deal with the uncertain constraints and objective function in (29) based on the theory of Linear Matrix Inequality (LMI). We will propose a semidefinite relaxation paradigm of the uncertain optimization model (29), which can be solved efficiently to result in a robust MPC control scheme.

\section{Closed-Loop Robust Control Design With Causal Disturbance FEedback}

The basic idea to realize the closed-loop control is to map the system state to the control sequence. A widely-adopted paradigm is the linear state feedback, which parametrizes the future control by linearly combining the predicted system states like $\boldsymbol{u}(k+j \mid k)=\boldsymbol{k} \boldsymbol{x}(k+j \mid k)+\boldsymbol{c}(k+j \mid k)(j=$ $0,1, \ldots, H-1)$, where the gain matrix $\boldsymbol{k}$ is usually designed off-line and $\boldsymbol{c}(k+j \mid k)$ is a new decision variable with the same dimension of $\boldsymbol{u}(k+j \mid k)$. However, parameterizing $\boldsymbol{u}(k+j \mid k)$ with respect to $\boldsymbol{x}(k+j \mid k)$ can lead to the nonlinearity in the formulation of (29), which, thus, may not be solved efficiently by using convex optimization methods [57], [60]. When carefully looking into the mathematical structure of (22), it can be found that the external disturbance term $\boldsymbol{D}$ and the predicted control $\boldsymbol{U}$ are two peer unknown variables. The past disturbances can affect the current state, such that the current control implicitly relies on the effect of the past disturbances. At this point, an alternative way to maintain the convexity in the closed-loop min-max MPC formulation is to parameterize the control signal of each vehicle directly with the disturbances via a causal structure [55], [56]. That is, we use a causal structure to realize the disturbance feedback as

$$
\boldsymbol{U}=\boldsymbol{K} \boldsymbol{D}+\boldsymbol{U}_{K},
$$

where $\boldsymbol{U}_{K}$ is a new decision variable, i.e., a column vector with the same size of $\boldsymbol{U}$. The gain matrix $\boldsymbol{K} \in \mathbb{R}^{H N \times 3 H N}$ has a causal structure designed as follows

$$
\boldsymbol{K}=\left(\mathbb{I}_{\text {tril }}^{H \times H} \otimes \mathbb{I}_{\text {full }}^{N \times 3 N}\right) \circ \boldsymbol{K}_{\text {full }}^{N H \times 3 N H},
$$

where $\mathbb{I}_{\text {tril }}^{H \times H}$ is a $H \times H$ lower tridiagonal matrix, the partial elements of which below the main diagonal are set to 1 while the others (i.e., those elements above as well as including the main diagonal) are $0 . \mathbb{I}_{\text {full }}^{N \times 3 N}$ is a $N \times 3 N$ real matrix, all of whose elements are 1 . Besides, $\boldsymbol{K}_{\text {full }}^{N H \times 3 N H}$ is a $N H \times$ $3 N H$ full real gain matrix. $\otimes$ is the Kronecker tensor product operator, and $\circ$ is the Hadamard product operator that gets the element-wise product of two compatible matrices.

We remark that the introduction of $\mathbb{I}_{\text {tril }}^{H \times H} \otimes \mathbb{I}_{\text {full }}^{N \times 3 N}$ in (31) can design a specific causal structure, in which each control sequence $\boldsymbol{u}(k+j \mid k)$ at the prediction step $j$ depends on all the past disturbance effects $\{\boldsymbol{d}(k+l \mid k), l=0,1, \ldots, j-1\}$. In this way, the feedback structure (30) parameterizes the platoon control sequence $\boldsymbol{U}$ directly in the external uncertainty term $D$. Substituting the causal disturbance feedback-based control design (30) into the constraints in the model (29), we can derive an equivalent epigraph formulation for the closed-loop min-max MPC as follows

$$
\begin{aligned}
& \min _{\boldsymbol{K}, \boldsymbol{U}_{K}, \gamma}: \gamma \\
& \text { s.t. }\left\{\begin{array}{l}
\boldsymbol{X}=\boldsymbol{G}_{A} \boldsymbol{x}(k \mid k)+\boldsymbol{G}_{B} \boldsymbol{U}_{K}+\left(\boldsymbol{G}_{B} \boldsymbol{K}+\boldsymbol{G}_{D}\right) \boldsymbol{D} \\
\boldsymbol{X}^{\mathrm{T}} \boldsymbol{W}_{X} \boldsymbol{X} \\
+\left(\boldsymbol{K} \boldsymbol{D}+\boldsymbol{U}_{K}\right)^{\mathrm{T}} \boldsymbol{W}_{U}\left(\boldsymbol{K} \boldsymbol{D}+\boldsymbol{U}_{K}\right) \leq \gamma, \forall \boldsymbol{D} \in \mathbb{D}, \\
\boldsymbol{X} \in \mathbb{X}, \forall \boldsymbol{D} \in \mathbb{D}, \\
\boldsymbol{K} \boldsymbol{D}+\boldsymbol{U}_{K} \in \mathbb{U}, \forall \boldsymbol{D} \in \mathbb{D}
\end{array}\right.
\end{aligned}
$$

Now, from both (30) and (32), it can be seen that the mathematical mapping from the decision variables $\boldsymbol{K}$ and $\boldsymbol{U}_{K}$ to the control sequence $\boldsymbol{U}$ and the system state $\boldsymbol{X}$ is bi-linear, respectively. This mathematical property is significant, since it guarantees the model convexity and thus enables legacy convex optimization techniques to come into play. 


\section{A. Robust Counterpart of Uncertain Control Constraint}

It is noted that the control constraint $\boldsymbol{U} \in \mathbb{U}$ incorporates the uncertainty $\boldsymbol{D}$ since we use the disturbance feedback in the control loop. Thus, to deal with the control bound constraint, we would like to derive its robust counterpart. Here, a lemma on the maximum value of a linear function constrained within the unit cube is first introduced as follows, while its proof is detailed in Appendix A.

Lemma 1: Given $\boldsymbol{C}$ is a real matrix with a compatible dimension with a column vector $\boldsymbol{x}$, we can have

$$
\max _{|\boldsymbol{x}| \leq 1}\{\boldsymbol{C} \boldsymbol{x}\} \leq|\boldsymbol{C}| \mathbf{1},
$$

where $|\boldsymbol{C}|$ is an element-wise absolute value matrix of $\boldsymbol{C} . \mathbf{1}$ is a column vector whose elements are all equal to 1 , and the dimension of $\mathbf{1}$ is as the same as that of $\boldsymbol{x}$.

Using Lemma 1 and recalling the box-constrained uncertainty set $\mathbb{D}$, the control bound constraint with the uncertainty $\boldsymbol{D}, \boldsymbol{U} \in \mathbb{U}$ for all $\boldsymbol{D} \in \mathbb{D}$, can be satisfied if the following inequality holds

$$
\left|\boldsymbol{A}_{U} \boldsymbol{K}\right| \mathbf{1}+\boldsymbol{A}_{U} \boldsymbol{U}_{K} \leq \boldsymbol{b}_{U}
$$

To deal with the absolute value of $\left|\boldsymbol{A}_{U} \boldsymbol{K}\right|$, we can introduce an additional matrix variable $\boldsymbol{\beta}_{U}$ and let

$$
\boldsymbol{A}_{U} \boldsymbol{K} \leq \boldsymbol{\beta}_{U}, \quad-\boldsymbol{A}_{U} \boldsymbol{K} \leq \boldsymbol{\beta}_{U} .
$$

Now, combining (35) and (34), we can further derive the robust counterpart of the uncertain control bound constraint as follows

$$
\left\{\begin{array}{l}
\boldsymbol{\beta}_{U} \mathbf{1}+\boldsymbol{A}_{U} \boldsymbol{U}_{K} \leq \boldsymbol{b}_{U} \\
\boldsymbol{A}_{U} \boldsymbol{K} \leq \boldsymbol{\beta}_{U} \\
-\boldsymbol{A}_{U} \boldsymbol{K} \leq \boldsymbol{\beta}_{U}
\end{array}\right.
$$

\section{B. Robust Counterpart of Uncertain State Constraint}

Using the same logic in Subsection IV-A, we can also derive the robust counterpart of the uncertain state bound constraint by introducing a new matrix variable $\boldsymbol{\beta}_{X}$. That is, substituting the disturbance feedback-based control (30) into (22), the constraint $\boldsymbol{X} \in \mathbb{X}, \forall \boldsymbol{D} \in \mathbb{D}$ is satisfied under the following condition

$$
\left\{\begin{array}{l}
\boldsymbol{\beta}_{X} \mathbf{1}+\boldsymbol{A}_{X}\left(\boldsymbol{G}_{A} \boldsymbol{x}(k \mid k)+\boldsymbol{G}_{B} \boldsymbol{U}_{K}\right) \leq \boldsymbol{b}_{X} \\
\boldsymbol{A}_{X}\left(\boldsymbol{G}_{B} \boldsymbol{K}+\boldsymbol{G}_{D}\right) \leq \boldsymbol{\beta}_{X} \\
-\boldsymbol{A}_{X}\left(\boldsymbol{G}_{B} \boldsymbol{K}+\boldsymbol{G}_{D}\right) \leq \boldsymbol{\beta}_{X} .
\end{array}\right.
$$

For simplicity, let $\boldsymbol{\beta}=\operatorname{col}\left\{\boldsymbol{\beta}_{X}, \boldsymbol{\beta}_{U}\right\}$. By integrating both the robust counterparts of the control and state constraints in (36) and (37), we can derive the following compact form as the linear system constraint $C_{1}$

$$
C_{1}:\left\{\begin{array}{l}
\boldsymbol{\beta} \mathbf{1}+\left[\begin{array}{c}
\boldsymbol{A}_{X}\left(\boldsymbol{G}_{A} \boldsymbol{x}(k \mid k)+\boldsymbol{G}_{B} \boldsymbol{U}_{K}\right) \\
\boldsymbol{A}_{U} \boldsymbol{U}_{K}
\end{array}\right] \leq\left[\begin{array}{l}
\boldsymbol{b}_{X} \\
\boldsymbol{b}_{U}
\end{array}\right], \\
{\left[\begin{array}{c}
\boldsymbol{A}_{X}\left(\boldsymbol{G}_{B} \boldsymbol{K}+\boldsymbol{G}_{D}\right) \\
\boldsymbol{A}_{U} \boldsymbol{K}
\end{array}\right] \leq \boldsymbol{\beta},} \\
-\left[\begin{array}{c}
\boldsymbol{A}_{X}\left(\boldsymbol{G}_{B} \boldsymbol{K}+\boldsymbol{G}_{D}\right) \\
\boldsymbol{A}_{U} \boldsymbol{K}
\end{array}\right] \leq \boldsymbol{\beta} .
\end{array}\right.
$$

\section{Robust Counterpart of Uncertain Performance Constraint}

To proceed, we introduce the well-known S-procedure and the Schur complement lemmas as follows.

Lemma 2 (The Schur Complement [65]): Given a positive definite real matrix $Q \succ 0$, the following inequality is equivalent for any positive semidefinite real matrix $\boldsymbol{P} \succeq 0$,

$$
\boldsymbol{P}-\boldsymbol{Z}^{\mathrm{T}} \boldsymbol{Q}^{-1} \boldsymbol{Z} \succeq 0 \Leftrightarrow\left[\begin{array}{cc}
\boldsymbol{P} & \boldsymbol{Z}^{\mathrm{T}} \\
\boldsymbol{Z} & \boldsymbol{Q}
\end{array}\right] \succeq 0
$$

where $\boldsymbol{Z}$ is any real matrix of an appropriate dimension.

Lemma 3 (The S-Procedure [66]): Let $\boldsymbol{F}_{l}=\boldsymbol{F}_{l}^{\mathrm{T}} \in \mathbb{R}^{m \times m}$, $l=0,1, \ldots, n$, be a sequence of symmetric real matrices, and $T_{l}(\boldsymbol{x})=\boldsymbol{x}^{\mathrm{T}} \boldsymbol{F}_{l} \boldsymbol{x}$ the corresponding quadratic functions with respect to $\boldsymbol{x} \in \mathbb{R}^{m \times 1}$. A sufficient condition for the following implication to hold

$$
\forall x \in \mathbb{R}^{m \times 1}, \quad T_{l}(\boldsymbol{x}) \geq 0, l=1,2, \ldots, n \Rightarrow T_{0}(\boldsymbol{x}) \geq 0
$$

is that there exist $\lambda=\operatorname{col}\left\{\lambda_{l} \geq 0, l=1,2, \ldots, n\right\}$ such that

$$
\boldsymbol{F}_{0}-\sum_{l=1}^{n} \lambda_{l} \boldsymbol{F}_{l} \geq 0 \text {. }
$$

Based on the Schur complement, we derive another equivalent form of the performance constraint in (29), $\boldsymbol{X}^{\mathrm{T}} \boldsymbol{W}_{X} \boldsymbol{X}+$ $\boldsymbol{U}^{\mathrm{T}} \boldsymbol{W}_{U} \boldsymbol{U} \leq \gamma, \forall \boldsymbol{D} \in \mathbb{D}$, as follows.

Theorem 1: Let $g\left(\boldsymbol{U}_{K}, \gamma\right) \in \mathbb{R}^{(1+3 H N+H N) \times(1+3 H N+H N)}$ denote the following matrix associated with $\gamma$ and $\boldsymbol{U}_{K}$

$$
g\left(\boldsymbol{U}_{K}, \gamma\right)=\left[\begin{array}{ccc}
\gamma & \boldsymbol{Y}^{\mathrm{T}} & \boldsymbol{U}_{K}^{\mathrm{T}} \\
\boldsymbol{Y} & \boldsymbol{W}_{X}^{-1} & \mathbf{0}_{3 H N \times H N} \\
\boldsymbol{U}_{K} & \mathbf{0}_{H N \times 3 H N} & \boldsymbol{W}_{U}^{-1}
\end{array}\right],
$$

where $\boldsymbol{Y}=\boldsymbol{G}_{A} \boldsymbol{x}(k \mid k)+\boldsymbol{G}_{B} \boldsymbol{U}_{K}$. Besides, let $\boldsymbol{L} \in$ $\mathbb{R}^{(1+3 H N+H N) \times 3 H N}$ and $\boldsymbol{R} \in \mathbb{R}^{3 H N \times(1+3 H N+H N)}$ denote the following two matrices, respectively,

$$
\left\{\begin{aligned}
\boldsymbol{L} & =\left[\begin{array}{c}
1 \\
\mathbf{0}_{3 H N \times 1} \\
\mathbf{0}_{H N \times 1}
\end{array}\right] \mathbf{1}_{3 H N \times 1}^{\mathrm{T}}, \\
\boldsymbol{R} & =\left[\mathbf{0}_{3 H N \times 1},\left(\boldsymbol{G}_{D}+\boldsymbol{G}_{B} \boldsymbol{K}\right)^{\mathrm{T}}, \boldsymbol{K}^{\mathrm{T}}\right] .
\end{aligned}\right.
$$

The uncertain disturbance $\boldsymbol{D}$ can be decomposed as the form

$$
\boldsymbol{D}=\boldsymbol{\Delta}^{\mathrm{T}} \mathbf{1}_{3 H N \times 1},
$$

where $\boldsymbol{\Delta} \in \mathbb{R}^{3 H N \times 3 H N}$ is a diagonal matrix whose diagonal elements are equal to those in $\boldsymbol{D}$, i.e., $\boldsymbol{\Delta}=\operatorname{diag}\{\boldsymbol{D}\}$. The quadratic performance constraint $\boldsymbol{X}^{\mathrm{T}} \boldsymbol{W}_{X} \boldsymbol{X}+\boldsymbol{U}^{\mathrm{T}} \boldsymbol{W}_{U} \boldsymbol{U} \leq$ $\gamma, \forall \boldsymbol{D} \in \mathbb{D}$ is equivalent to

$$
g\left(\boldsymbol{U}_{K}, \gamma\right)+\boldsymbol{L} \boldsymbol{\Delta} \boldsymbol{R}+\boldsymbol{R}^{\mathrm{T}} \boldsymbol{\Delta}^{\mathrm{T}} \boldsymbol{L}^{\mathrm{T}} \succeq 0, \quad \forall \boldsymbol{\Delta} \in \mathcal{W},
$$

where $\mathcal{W}$ is similar to $\mathbb{D}$, i.e., $\mathcal{W}=\{\boldsymbol{\Delta}:|\boldsymbol{\Delta}| \leq 1\}$.

Following Theorem 1, we further derive another result that transforms the uncertain performance constraint into a robust counterpart, i.e., a mathematically-tractable LMI form.

Theorem 2: If there exist a sequence of positive real numbers $\lambda_{l} \in \mathbb{R}_{+}, l=1,2, \ldots, 3 H N$, such that

$$
\left[\begin{array}{cc}
g\left(\boldsymbol{U}_{K}, \gamma\right)-\boldsymbol{L} \boldsymbol{S}(\boldsymbol{\lambda}) \boldsymbol{L}^{\mathrm{T}} & \boldsymbol{R}^{\mathrm{T}} \\
\boldsymbol{R} & \boldsymbol{S}(\boldsymbol{\lambda})
\end{array}\right] \succeq 0
$$


holds where $\lambda=\operatorname{col}\left\{\lambda_{l}, l=1,2, \ldots, 3 H N\right\}$ and $\boldsymbol{S}(\lambda)=$ $\operatorname{diag}\{\lambda\}$, the uncertain LMI in (45) always holds for $\Delta \in \mathcal{W}$.

The proofs of Theorems 1 and 2 are given in Appendices B and $\mathrm{C}$, respectively. Besides, notice that we have

$$
\boldsymbol{L S}(\boldsymbol{\lambda}) \boldsymbol{L}^{\mathrm{T}}=\left[\begin{array}{ccc}
\mathbf{1}_{3 H N \times 1}^{\mathrm{T}} \boldsymbol{\lambda} & \mathbf{0}_{1 \times 3 H N} & \mathbf{0}_{1 \times H N} \\
\mathbf{0}_{3 H N \times 1} & \mathbf{0}_{3 H N \times 3 H N} & \mathbf{0}_{3 H N \times H N} \\
\mathbf{0}_{H N \times 1} & \mathbf{0}_{H N \times 3 H N} & \mathbf{0}_{H N \times H N}
\end{array}\right] .
$$

Substituting (47) into the results of Theorems 1 and 2, we can further present the robust counterpart of the quadratic performance constraint in a LMI form $C_{2}$ as follows

$$
C_{2}:\left[\begin{array}{cccc}
\gamma-\mathbf{1}_{3 H N \times 1}^{\mathrm{T}} \boldsymbol{\lambda} & \boldsymbol{Y}^{\mathrm{T}} & \boldsymbol{U}_{K}^{\mathrm{T}} & \mathbf{0}_{1 \times 3 H N} \\
\boldsymbol{Y} & \boldsymbol{W}_{X}^{-1} & \mathbf{0}_{3 H N \times H N} & \boldsymbol{G}_{K} \\
\boldsymbol{U}_{K} & \mathbf{0}_{H N \times 3 H N} & \boldsymbol{W}_{U}^{-1} & \boldsymbol{K} \\
\mathbf{0}_{3 H N \times 1} & \boldsymbol{G}_{K}^{\mathrm{T}} & \boldsymbol{K}^{\mathrm{T}} & \boldsymbol{S}(\boldsymbol{\lambda})
\end{array}\right] \succeq 0 .
$$

\section{Closed-Loop Robust Control Design}

Using the robust counterparts of the state and control constraints and the performance constraint, $C_{1}$ and $C_{2}$, we finally derive a robust counterpart model of (32) for designing the closed-loop platoon control as follows

$$
\begin{aligned}
\min _{\gamma, \boldsymbol{K}, \boldsymbol{U}_{K}, \boldsymbol{\beta}, \lambda}: \gamma \\
\text { s.t. } C_{1} \text { and } C_{2} .
\end{aligned}
$$

It is seen from (49) that the semidefinite programming model is a tractable convex optimization model. It can be effectively solved by using some convex optimization techniques such as the interior-point method and its various variants [66]. Based on solving the model (49), we can design the robust platoon control algorithm as detailed in Algorithm 1. The implementation of the proposed control is also illustrated in Figure 1, which follows a centralized computation paradigm. In the implementation framework, Vehicle 1, the first platooning vehicle, is selected as a master node for the coordination of the vehicle platoon. It takes responsibility for solving the closed-loop min-max MPC platoon model (49) and distributing the generated control to the other following vehicles via vehicular wireless communications.

It is remarked that, since the coverage of one-hop vehicular communication and the radio resources are usually limited in reality, the number of vehicles supported by an inter-connected platoon is not too large. For example, a proper platoon size ranges from 5 to 10 in a real application scenario. Thus, the complexity of (49) can be effectively coped with the centralized computation framework. Nevertheless, a distributed MPC framework may be more appealing when considering to deploy the platoon control in a large-scale system. Some related works can be found in [46], [47], [62], while the extension of the proposed closed-loop min-max MPC to a distributed computation scenario is left as our future work.

\section{RECURSIVE FEASIBILITY AND ISPS STABILITY}

Let $\boldsymbol{k}_{x} \in \mathbb{R}^{N \times 3 N}$ be a $N \times 3 N$ real matrix and $\boldsymbol{K}_{X}=$ $\operatorname{diag}\left\{\boldsymbol{k}_{x}, j=0,1, \ldots, H-1\right\}$. In fact, because a disturbance

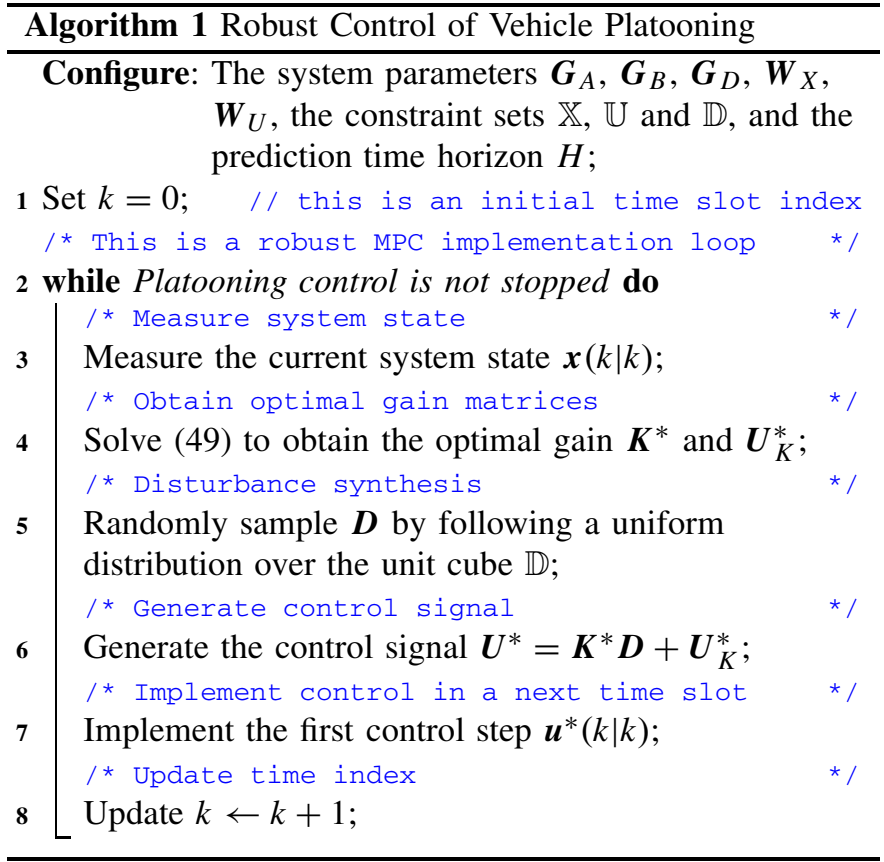

feedback control can be equivalent to a state feedback control, the gain matrix $\boldsymbol{k}_{x}$ always exists and is determined by enforcing the relationship $\boldsymbol{K}_{X} \boldsymbol{X}=\boldsymbol{K}^{*} \boldsymbol{D}+\boldsymbol{U}_{K}^{*}$ where $\boldsymbol{K}^{*}$ and $\boldsymbol{U}_{K}^{*}$ are obtained from (49). Due to the positive definiteness and symmetry of $\boldsymbol{w}_{x}$ and $\boldsymbol{w}_{u}$, there correspondingly exist two positive definite symmetric matrices $\boldsymbol{P}_{x}$ and $\boldsymbol{P}_{u}$ such that $\boldsymbol{w}_{x}$, $\boldsymbol{w}_{u}$ can be re-formulated as $\boldsymbol{w}_{x}=\boldsymbol{P}_{x}^{\mathrm{T}} \boldsymbol{P}_{x}$ and $\boldsymbol{w}_{u}=\boldsymbol{P}_{u}^{\mathrm{T}} \boldsymbol{P}_{u}$, respectively. Besides, the positive definiteness and symmetry of $\boldsymbol{V}$ can also guarantee the existence of its inverse, $\boldsymbol{V}^{-1}$. Using the above notations, we introduce the following lemma.

Lemma 4: There exists a robust positively invariant (RPI) set depending on the terminal weight $\boldsymbol{V}$ for the model (10), $\mathcal{E}(\boldsymbol{V})$, such that $\mathcal{E}(\boldsymbol{V}) \subseteq \mathcal{X}$ and $\mathbf{0}_{3 N \times 1} \in \operatorname{int}(\mathcal{E}(\boldsymbol{V}))$.

The proof of Lemma 4 is provided in Appendix D. According the property of the RPI set $\mathcal{E}(\boldsymbol{V})$, we can further have $\boldsymbol{x}(k+i+1 \mid k) \in \mathcal{E}(\boldsymbol{V})$ for all $i=0,1, \ldots, H-1, \boldsymbol{x}(k+i \mid k) \in$ $\mathcal{E}(\boldsymbol{V})$ and $\boldsymbol{d}(k+i \mid k) \in \mathcal{D}$. Let $\boldsymbol{M}_{K}=\boldsymbol{M}_{A}+\boldsymbol{M}_{B} \boldsymbol{k}_{x}$. We obtain the result on the recursive feasibility and ISpS.

Theorem 3: Algorithm 1 is recursively feasible by enforcing $\mathcal{E}(\boldsymbol{V})$ as a terminal state constraint set, and the model (10) in closed-loop with the min-max MPC control (49) is ISpS in $\mathcal{E}(\boldsymbol{V})$ if there exists a positive real number $\alpha>0$ such that the following LMI holds

$\left[\begin{array}{ccccc}\boldsymbol{V} & \mathbf{0}_{3 N \times 3 N} & \boldsymbol{M}_{K}^{\mathrm{T}} & \boldsymbol{P}_{x}^{\mathrm{T}} & \boldsymbol{k}_{x}^{\mathrm{T}} \boldsymbol{P}_{u}^{\mathrm{T}} \\ \mathbf{0}_{3 N \times 3 N} & \alpha \boldsymbol{M}_{D}^{\mathrm{T}} \boldsymbol{M}_{D} & \boldsymbol{M}_{D}^{\mathrm{T}} & \mathbf{0}_{3 N \times 3 N} & \mathbf{0}_{3 N \times N} \\ \boldsymbol{M}_{K} & \boldsymbol{M}_{D} & \boldsymbol{V}^{-1} & \mathbf{0}_{3 N \times 3 N} & \mathbf{0}_{3 N \times N} \\ \boldsymbol{P}_{x} & \mathbf{0}_{3 N \times 3 N} & \mathbf{0}_{3 N \times 3 N} & \boldsymbol{I}_{3 N \times 3 N} & \mathbf{0}_{3 N \times N} \\ \boldsymbol{P}_{u} \boldsymbol{k}_{x} & \mathbf{0}_{N \times 3 N} & \mathbf{0}_{N \times 3 N} & \mathbf{0}_{N \times 3 N} & \boldsymbol{I}_{N \times N}\end{array}\right] \succeq 0$.

Appendix E details the proof of Theorem 3 above. From this theorem, the recursive feasibility and the ISpS of the perturbed platoon system (10) depend on the configuration of the proposed min-max MPC model, $\boldsymbol{w}_{x}, \boldsymbol{w}_{u}$ and $\boldsymbol{V}$, and the construction of the RPI terminal set $\mathcal{E}(\boldsymbol{V})$. It is remarked that these matrices can be determined off-line by empirical 
TABLE I

PARAMETER SETTINGS

\begin{tabular}{ll}
\hline Symbol & Value \\
\hline$N$ & 5 \\
$\Delta t$ & $1.5 \mathrm{~s}$ \\
$l_{i}$ & $5 \mathrm{~m}$ \\
$\kappa$ & 0.9 \\
$\tau$ & $0.01 \mathrm{~s}$ \\
$\tau_{s}$ & $0.1 \mathrm{~s}$ \\
$H$ & 10 \\
$\boldsymbol{w}_{x}$ & $\boldsymbol{I}_{5 \times 5} \otimes \operatorname{diag}\{10,1,0.1\}$ \\
$\boldsymbol{w}_{u}$ & $\boldsymbol{I}_{5 \times 5} \otimes \operatorname{diag}\{0.01\}$ \\
$\boldsymbol{V}$ & $\boldsymbol{I}_{5 \times 5} \otimes \operatorname{diag}\left\{[0.3288,5.3829,0.6466] \times 10^{4}\right\}$ \\
$\boldsymbol{M}_{D}$ & $0.7 \boldsymbol{I}_{15 \times 15}$ \\
$\boldsymbol{x}^{-}$ & $\operatorname{col}\left\{[0,-5,-3]^{\mathrm{T}}, \forall i=1,2, \ldots, 5\right\}$ \\
$\boldsymbol{x}^{+}$ & $\operatorname{col}\left\{[\infty, 5,3]^{\mathrm{T}}, \forall i=1,2, \ldots, 5\right\}$ \\
$\boldsymbol{u}^{-}$ & $\operatorname{col}\{-5, \forall i=1,2, \ldots, 5\}$ \\
$\boldsymbol{u}^{+}$ & $\operatorname{col}\{5, \forall i=1,2, \ldots, 5\}$ \\
\hline &
\end{tabular}

analysis based on checking whether they can satisfy the condition (50) in Theorem 3. $\mathcal{E}(\boldsymbol{V})$ can also be constructed by using the homothetic transformation approach [62] or the outer approximation approach [67]. In our system model, one simple type of $\mathcal{E}(\boldsymbol{V})$ can be chosen as an ellipsoid with a parameter $\rho_{x}$, i.e., letting $\mathcal{E}(\boldsymbol{V})=\left\{\boldsymbol{x} \in \mathcal{X}: \boldsymbol{x}^{\mathrm{T}} \boldsymbol{V} \boldsymbol{x} \leq \rho_{x}^{2}\right\}$ where $\rho_{x}^{2}$ must exist and relies on the boundedness of $\boldsymbol{x}$.

\section{PERformance Evaluation}

To conduct simulations, we consider a specific platoon system with 5 actual vehicles (indexed from 1 to 5 ) and a virtual vehicle as the leading reference (indexed by 0 ). In the simulations, Algorithm 1 is implemented by jointly using the MATLAB optimization modeling toolbox, YALMIP [68], [69], and the general optimization solver, MOSEK [70], to solve the proposed min-max MPC model (49). The system parameters are given in Table I. Additionally, the simulation time is set to 30 seconds. The trajectory profile of the virtual leading vehicle is shown in Figure 2, which is treated as the reference information for the first actual vehicle in the platoon. The virtual leader is simulated to uniformly accelerate within the first 6 seconds and then move at a constant velocity $v_{\text {desired }}=$ $60 \mathrm{~km} / \mathrm{h}$ after the uniform acceleration stage. The initial longitudinal positions of all the following vehicles are randomly generated within $[0,500](\mathrm{m})$ with the inter-vehicle spacing of at least $30 \mathrm{~m}$. The longitudinal velocity and acceleration of the vehicles are initialized to zero. The velocity bounds are specified as $v_{\min }=0 \mathrm{~m} / \mathrm{s}$ and $v_{\max }=120 / 3.6 \mathrm{~m} / \mathrm{s}$ for all the vehicles.

\section{A. Performance Verification}

In Figure 3, we show the control inputs generated by our robust MPC platoon controller. As can be seen, all the control inputs $\left\{u_{i}(t), i=1,2, \ldots, 5\right\}$ are strictly bounded within the specified interval $[-5,5]\left(\mathrm{m} / \mathrm{s}^{3}\right)$ even though they are time-varying in response to the external disturbances. Figure 4 illustrates the longitudinal position, velocity, and acceleration
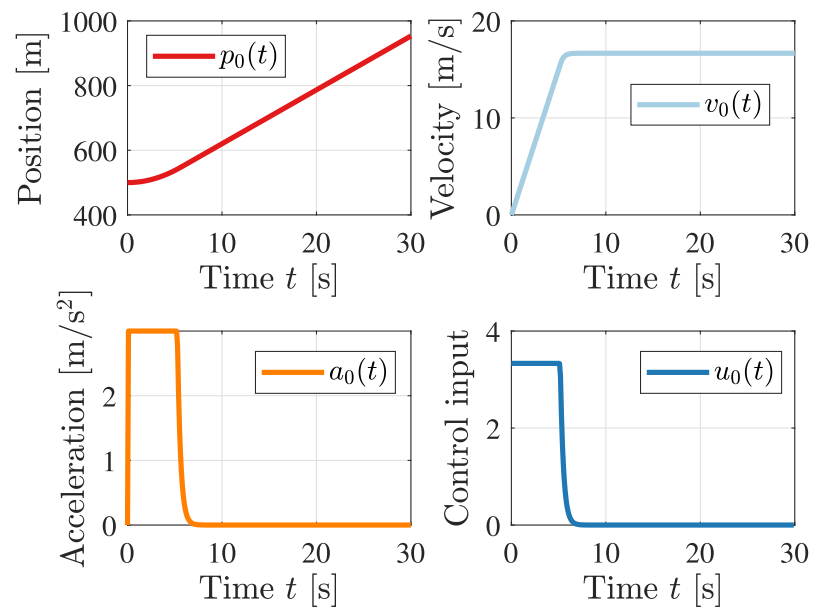

Fig. 2. The longitudinal position, velocity, acceleration, and control profiles of a virtual leading vehicle (i.e., $i=0$ ), which are treated as the reference signals for the first actual platooning vehicle, i.e., vehicle $i=1$.

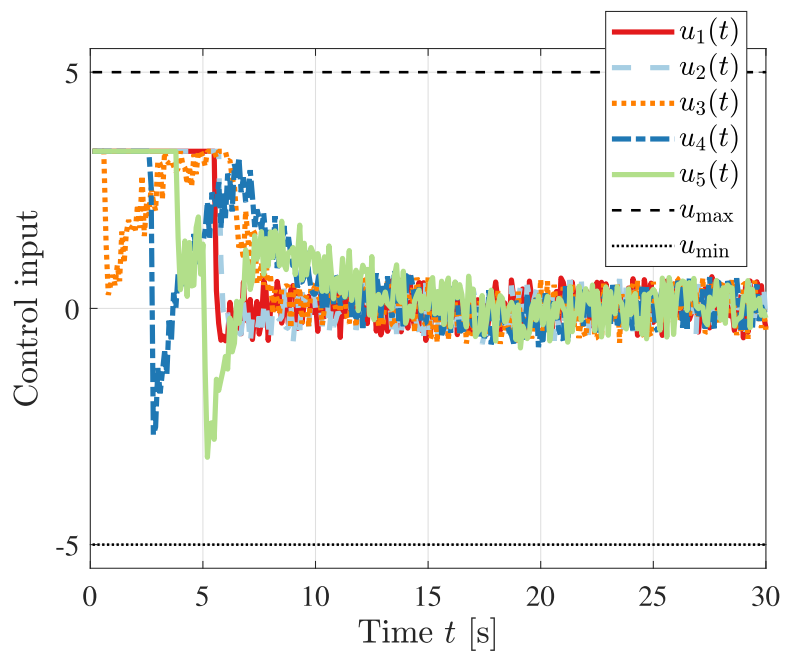

Fig. 3. The control inputs of the vehicles with the designed robust MPC platoon controller based on the causal disturbance feedback.

of the platooning vehicles under the robust MPC. It is seen that the vehicles can stably form a platoon without any chain collisions even in the presence of external disturbances. In particular, the vehicles can stably track the desired velocity $v_{\text {desired }}$ after about $15 \mathrm{~s}$. Their accelerations can always satisfy the bounds $[-3,3]\left(\mathrm{m} / \mathrm{s}^{2}\right)$ even under disturbances.

In Figures 5 and 6 , we show the position and velocity tracking errors between any two successive vehicles in the platoon. It is found from Figure 5 that all the position tracking errors can be reduced to near zero after about $6 \mathrm{~s}$. The root mean squared error (RMSE) of the position tracking errors $\left\{p_{i-1}(t)-p_{i}(t)-\left(v_{i}(t) \Delta t+l_{i-1}\right), i=1,2, \ldots, 5\right\}$ during the last $15 \mathrm{~s}$ is about $0.083692 \mathrm{~m}$. More importantly, the perturbed platooning vehicles can always guarantee their position errors above the allowable lower bound $\Delta p^{-}=0 \mathrm{~m}$. This indicates that the inter-vehicle spacing is always positive and thus the chain collisions are strictly avoided. From Figure 6, we can observe that the designed MPC platoon controller can also robustly stabilize the platooning vehicles. Their velocity 

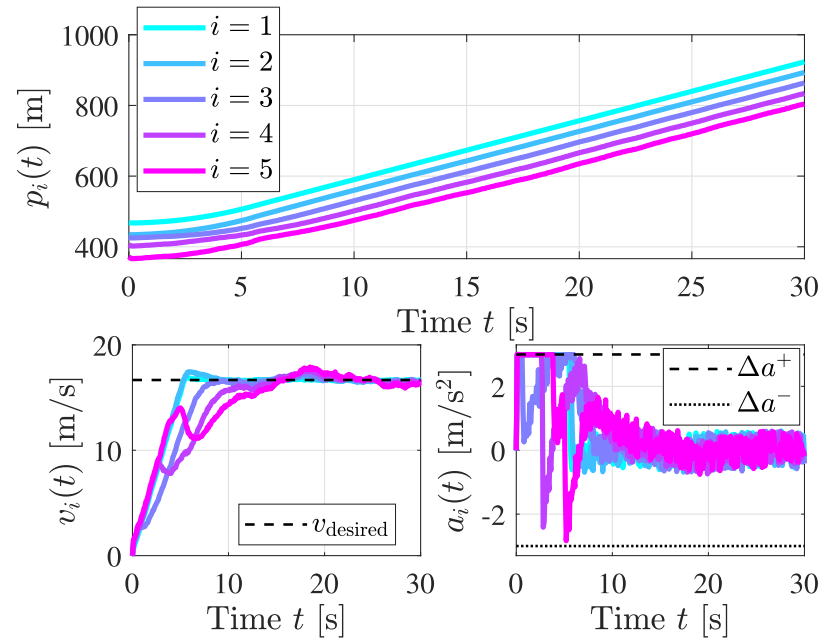

Fig. 4. The longitudinal position, velocity, and acceleration profiles of the vehicles with the designed robust MPC platoon controller based on the causal disturbance feedback.

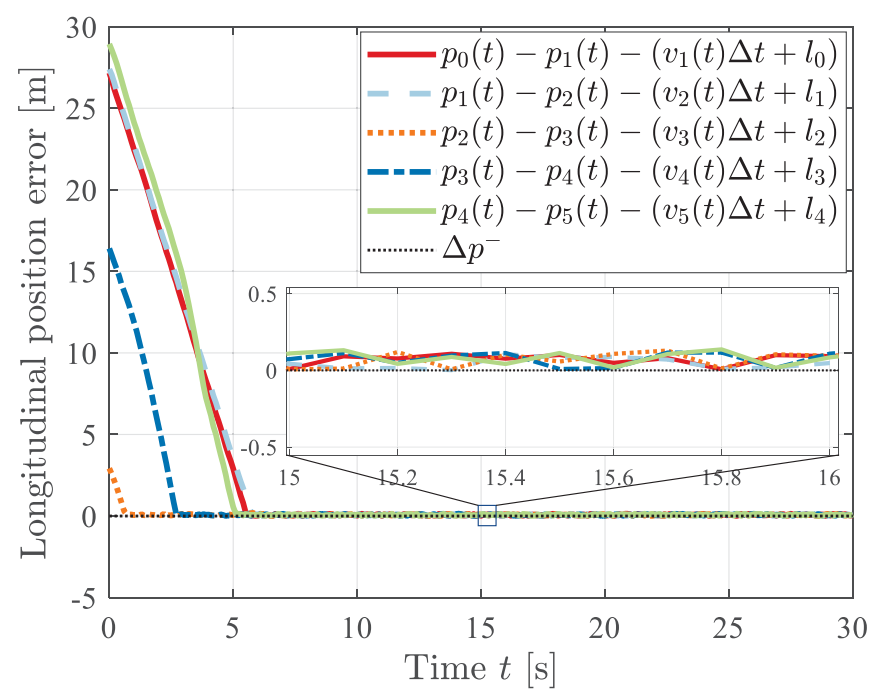

Fig. 5. The longitudinal position errors of the vehicles with the designed robust MPC platoon controller based on the causal disturbance feedback.

tracking errors are reduced to around zero, which have the RMSE of about $0.13841 \mathrm{~m} / \mathrm{s}$ during the last $15 \mathrm{~s}$. Even though they experience a relatively large error within $[0,10](s)$, their velocity tracking errors can be always bounded within the feasible interval $[-5,5](\mathrm{m} / \mathrm{s})$ and converge to a sufficiently small level after about $15 \mathrm{~s}$.

\section{B. Performance Comparison}

To show the advantage of the proposed platoon control method, we further conduct simulations with different types of external disturbances. Specifically, the disturbance coefficient matrix $\boldsymbol{M}_{D}$ is set to $0.5 \boldsymbol{I}_{5 \times 5} \otimes \operatorname{diag}\{[0,0,1]\}$ (Type I), $0.5 \boldsymbol{I}_{5 \times 5} \otimes \operatorname{diag}\{[0,1,1]\}$ (Type II), and $0.5 \boldsymbol{I}_{5 \times 5} \otimes$ $\operatorname{diag}\{[1,1,1]\}$ (Type III), respectively, which can simulate three types of external disturbances. In the Type I, the external disturbance term only appears in the dynamics of each vehicle's acceleration variation, $\dot{a}_{i}(t)$. In the type II, the external

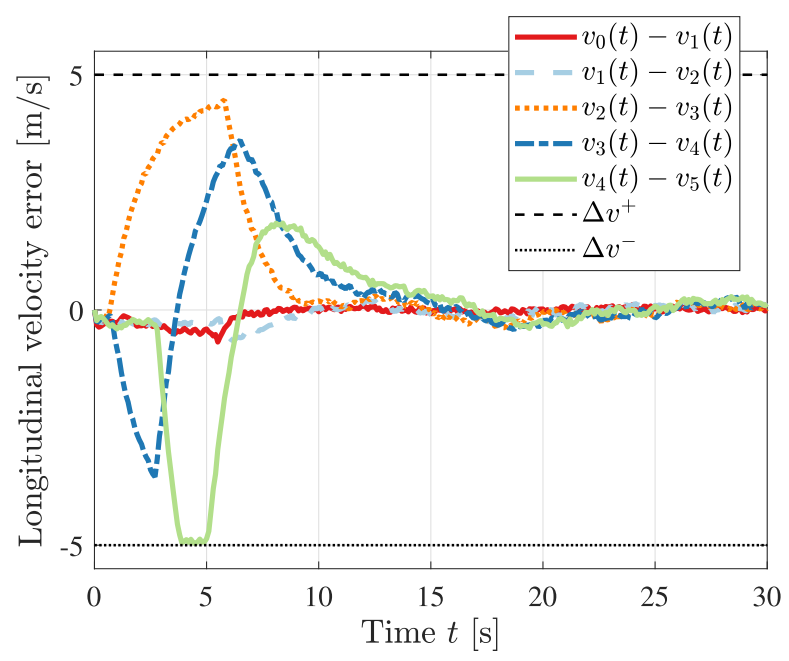

Fig. 6. The longitudinal velocity errors of the vehicles with the designed robust MPC platoon controller based on the causal disturbance feedback.

disturbance directly affects both of the velocity and acceleration variations, $\dot{v}_{i}(t)$ and $\dot{a}_{i}(t)$. In the type III, the external disturbance affects the dynamics of the position, velocity and acceleration variations, $\dot{p}_{i}(t), \dot{v}_{i}(t)$ and $\dot{a}_{i}(t)$. The other simulation parameters are set as in Table I. We compare our proposed method with other two conventional approaches, i.e., the nominal MPC-based and the Linear Quadratic Gaussian (LQG)-based platoon controllers. The nominal MPC approach is implemented without considering the external disturbance term, while the LQG controller is realized by integrating a linear quadratic regulator and a Kalman filter based on the state feedback. In the nominal MPC approach and the LQG approach, the simulation parameters are configured as in Table I. Additionally, the white process noise and measurement covariances of the Kalman filter in the LQG platoon controller are specified to $\boldsymbol{I}_{15 \times 15}$ and $10^{-6} \boldsymbol{I}_{15 \times 15}$, respectively. The simulation results are shown in Figures 7 to 9.

In Figure 7, it is obviously seen that increasing the effect of the external disturbance can result in a larger perturbation amplitude in the position tracking error $e_{1, i}(t)$. That is, the perturbation amplitude of the position tracking error with the disturbance type III is much larger than that with the disturbance types I and II. However, the perturbation amplitude of the position tracking error under our proposed robust MPC platoon control (marked by Robust CDF-MPC) is much smaller than that by the nominal MPC and the LQG controllers. In particular, from the sub-figures in Figure 7, our position tracking errors under different types of external disturbances are always positive, i.e., ensuring $e_{1, i}(t) \geq \Delta p^{-}$ for all $i$. This indicates that the inter-spacing of the vehicles can strictly ensure the platoon safety even when the system is perturbed all the time. By comparison, the position tracking errors of both the nominal MPC controller and the LQG controller are fluctuating around $\Delta p^{-}$, which means that these two control approaches cannot satisfy the state constraint.

Similarly, from Figure 8, it is observed that the perturbation amplitude in the velocity tracking error $e_{2, i}(t)$ can also be 

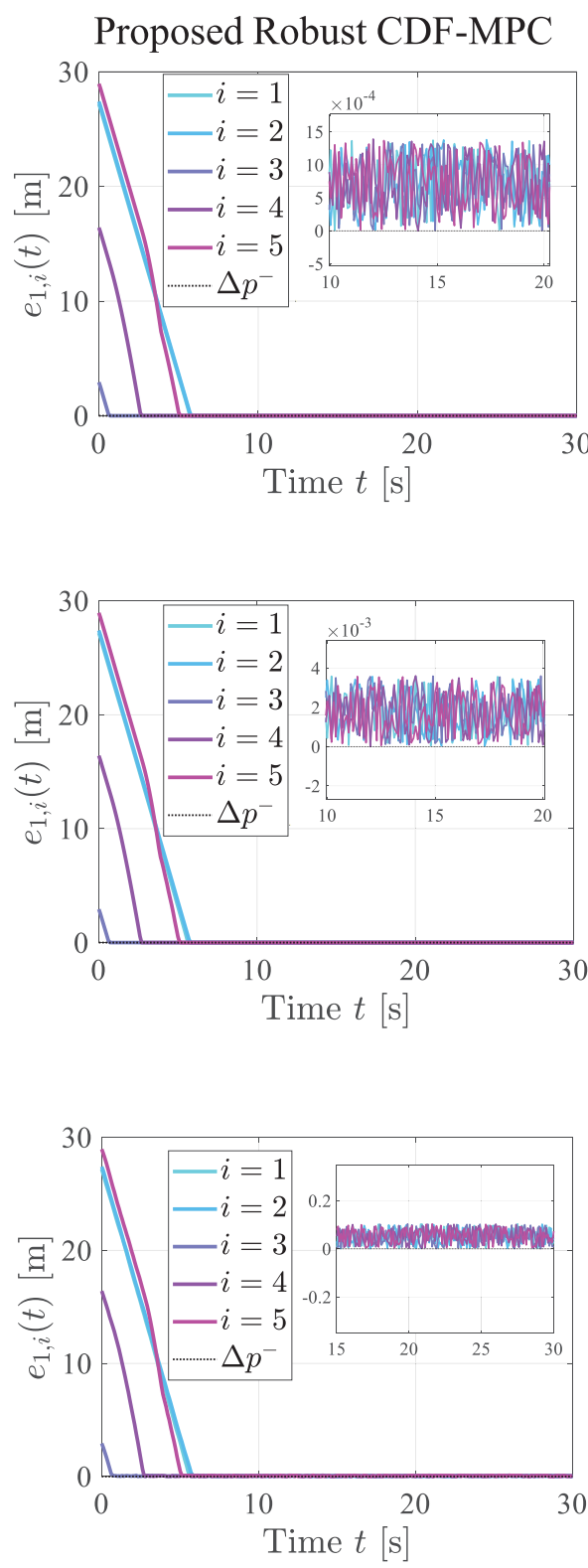

Nominal MPC Controller

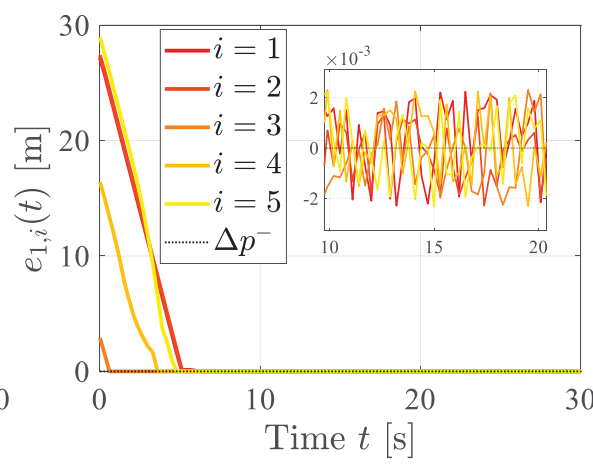

(a) Disturbance Type I

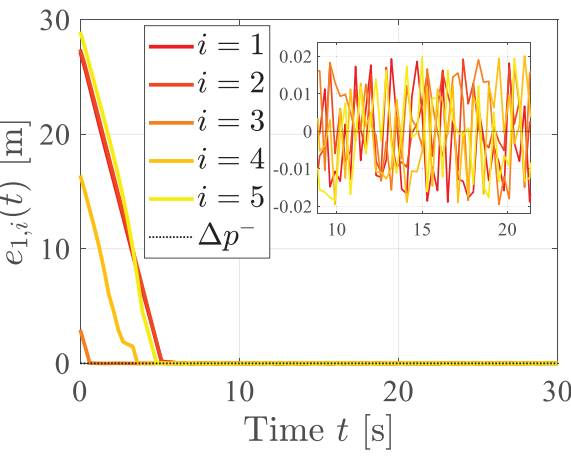

(b) Disturbance Type II

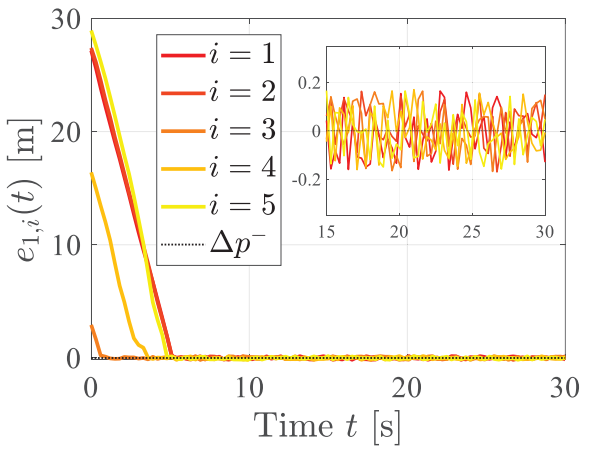

(c) Disturbance Type III
LQG Controller
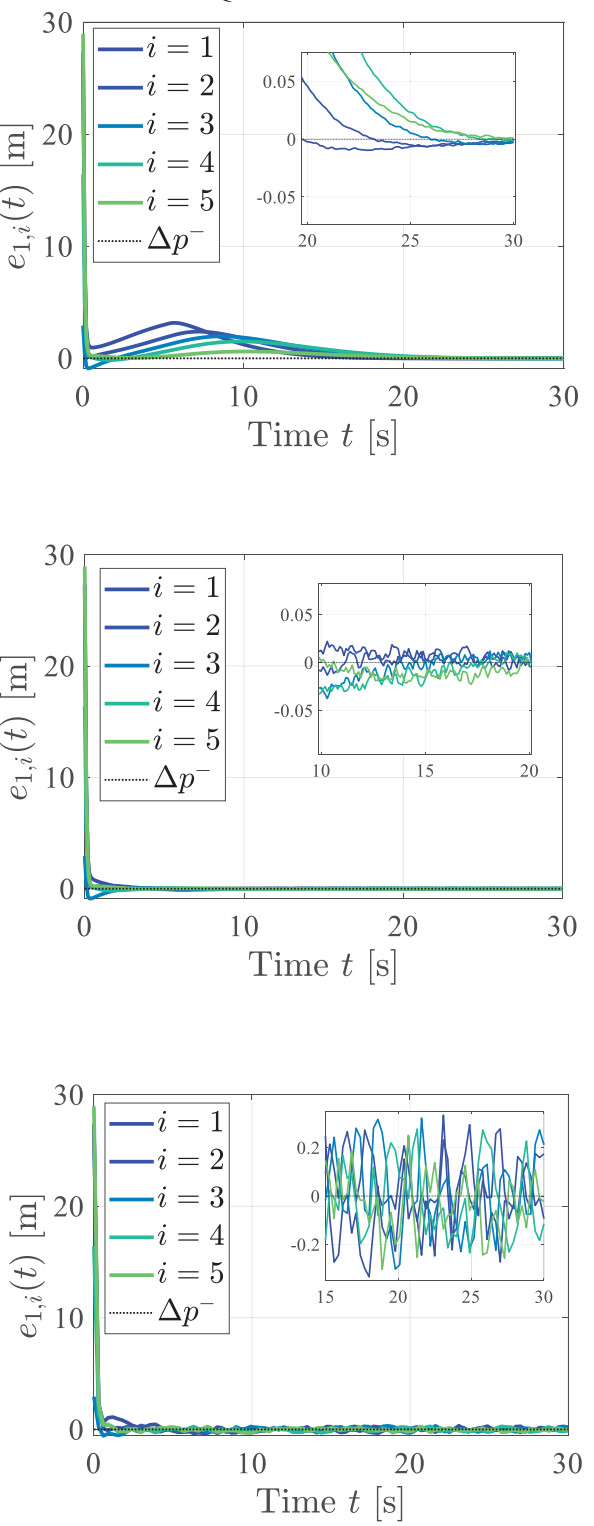

Fig. 7. The longitudinal position errors of the vehicles with different platoon controllers under different disturbances.

enhanced by increasing the disturbance intensity. Nonetheless, our robust MPC control can achieve the best stabilization performance under different types of external disturbances. In particular, even with the disturbance type III, our method can drive the velocity tracking error $e_{2, i}(t)$ to converge to almost zero and always guarantee $\Delta v^{-} \leq e_{2, i}(t) \leq \Delta v^{+}$for all $i$. A larger error perturbation is experienced by the nominal MPC controller during the last $15 \mathrm{~s}$. The LQG controller cannot ensure the constraint satisfaction during the first $10 \mathrm{~s}$.

From Figure 9, it is also seen that the acceleration profiles of all the platooning vehicles using the proposed control, $a_{i}(t)$, can always be bounded within the allowable interval $\left[\Delta a^{-}, \Delta a^{+}\right]\left(\mathrm{m} / \mathrm{s}^{2}\right)$. With the proposed MPC platoon control, the vehicles' accelerations can converge to a sufficiently small domain centered at zero. By contrast, the nominal MPC has a relatively large perturbation amplitude in the acceleration profiles during the last $10 \mathrm{~s}$. With the LQG control, the platooning vehicles have an unreasonable acceleration perturbation during the first $5 \mathrm{~s}$. Namely, the LQG control cannot satisfy the acceleration bounds under external uncertain disturbances.

In addition, as the proposed min-max MPC control method and the nominal MPC method are based on a constrained optimization technique, we compare their execution efficiency in terms of the computation time. Both the optimization-based methods are implemented on a single computer with the specific hardware conditions: Intel(R) Core(TM) i7-8750H CPU @ 2.20GHz-2.21GHz and RAM 8.00 GB. Monte Carlo simulations of both the control methods have been performed with 100 replications per disturbance situation aforementioned. The average computation time and the corresponding standard 

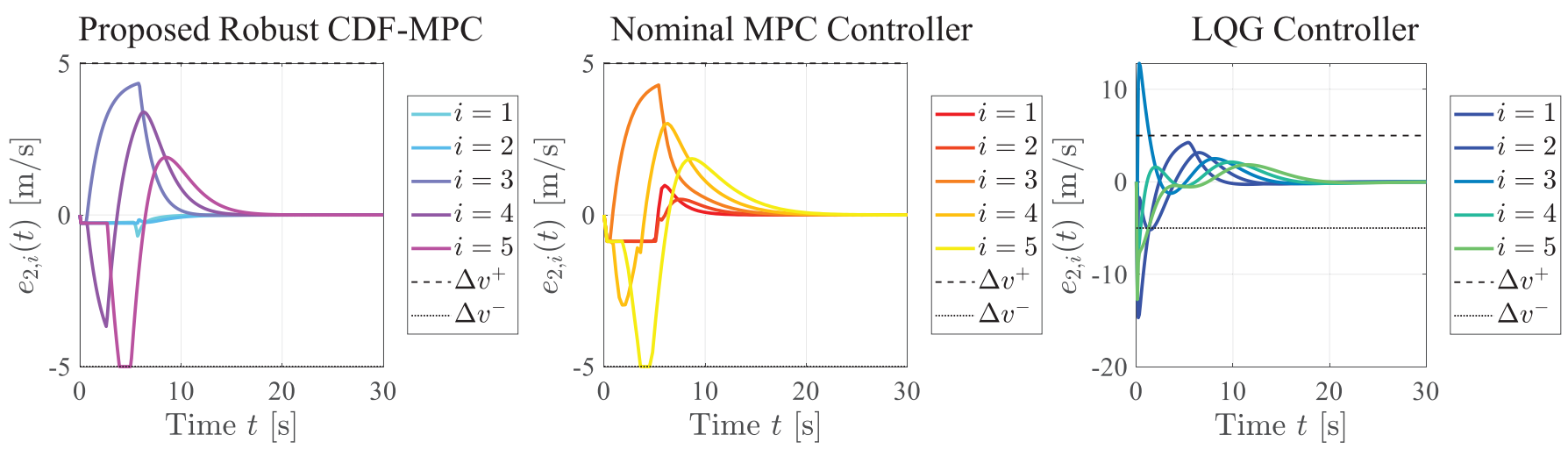

(a) Disturbance Type I
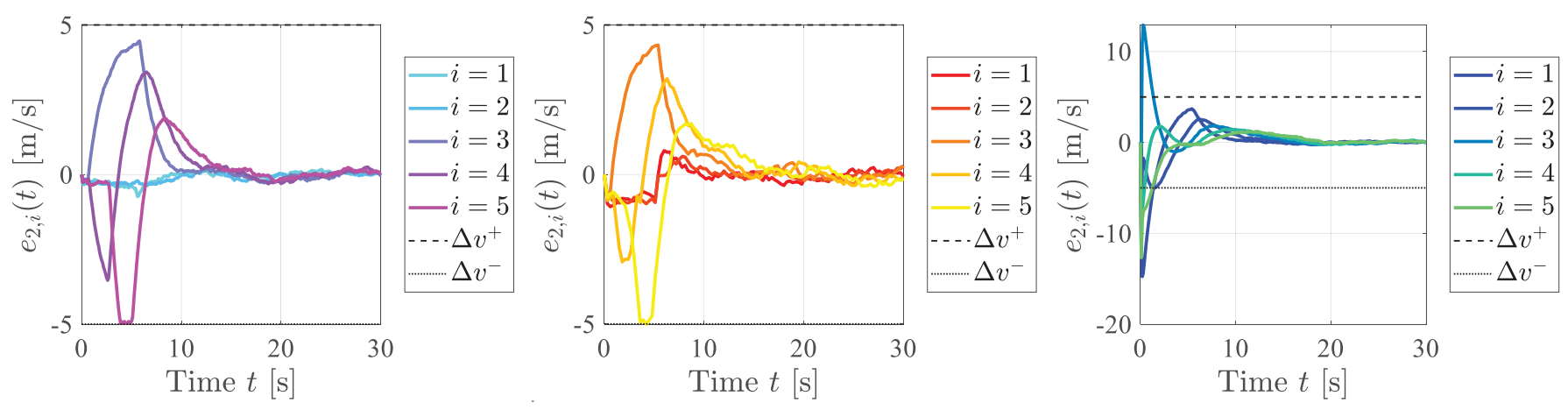

(b) Disturbance Type II
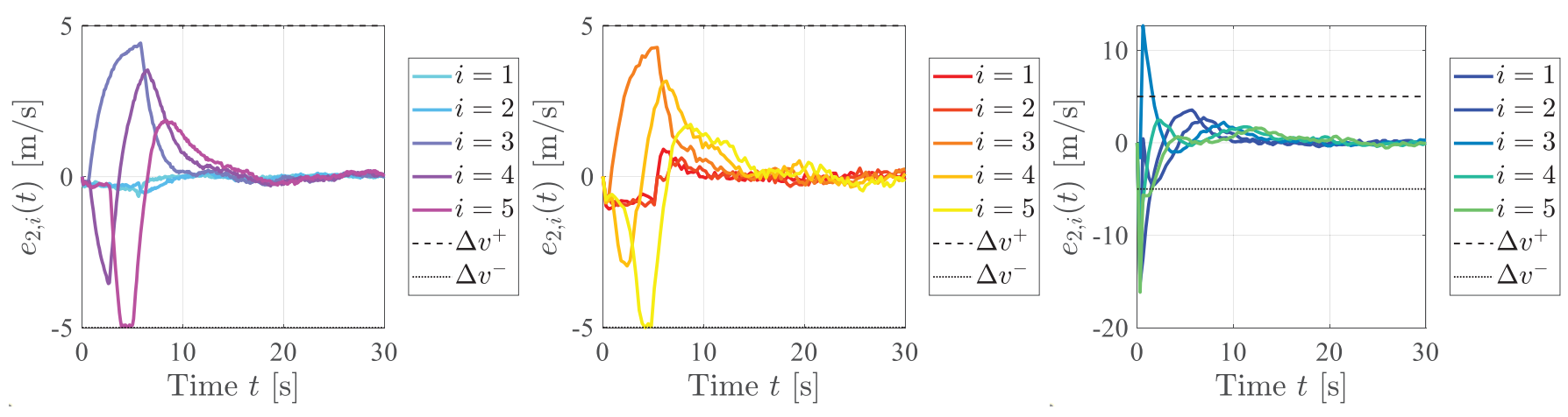

(c) Disturbance Type III

Fig. 8. The longitudinal velocity errors of the vehicles with different platoon controllers under different disturbances.

TABLE II

The Mean and STANdard DeViation OF Computation Time For OUR Proposed Robust MPC Method and the Nominal MPC Method

\begin{tabular}{lccccc}
\hline & \multicolumn{2}{c}{ Our robust MPC } & & \multicolumn{2}{c}{ Nominal MPC } \\
\cline { 2 - 3 } \cline { 5 - 6 } Disturbance & Avg. [ms] & Std. [ms] & & Avg. [ms] & Std. [ms] \\
\hline Type I & 38.928 & 1.3754 & & 3.7775 & 0.38494 \\
Type II & 37.738 & 1.5185 & & 0.69397 & $6.2915 \times 10^{-2}$ \\
Type III & 38.306 & 1.5253 & & 0.71377 & $8.7126 \times 10^{-2}$ \\
\hline
\end{tabular}

deviation under different control methods and disturbance situations are summarized in Table II. By comparison, the computation time taken by our robust MPC method per control execution is about $38 \mathrm{~ms}$ on average under different types of external disturbances, which is higher than that of the nominal MPC method. This is logical and expected, since the computational complexity of the robust min-max MPC method is higher than that of the conventional MPC method. The main reason is that the robust min-max MPC needs to additionally solve the LMIs in a semidefinite programming problem in order to deal with the external uncertain disturbances involved in the system model. Nevertheless, it is also noticed that the computation time of the robust min-max MPC method is lower than the sample time $(100 \mathrm{~ms})$ configured for model discretization. This implies that the execution efficiency of the robust method is satisfactory and the robust control can be practically realized for actual real-time computation.

Finally, to further verify the superior performance of our proposed method in terms of system robustness, we conduct 

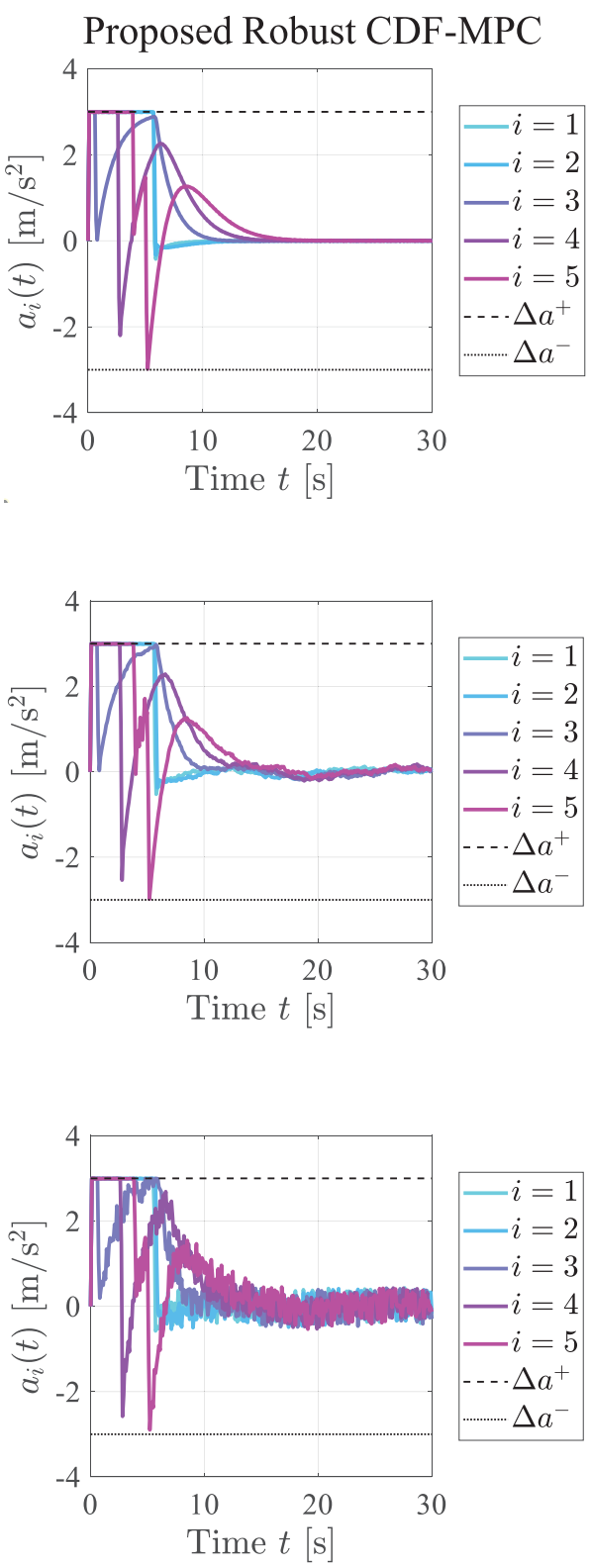

Nominal MPC Controller

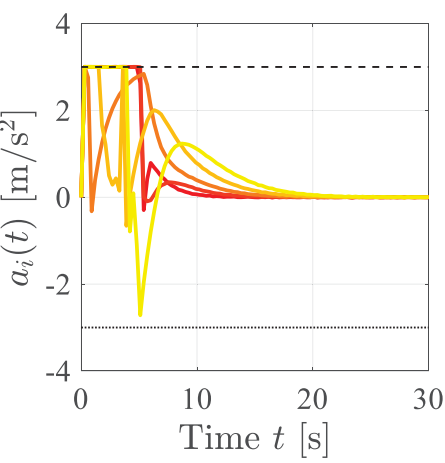

(a) Disturbance Type I
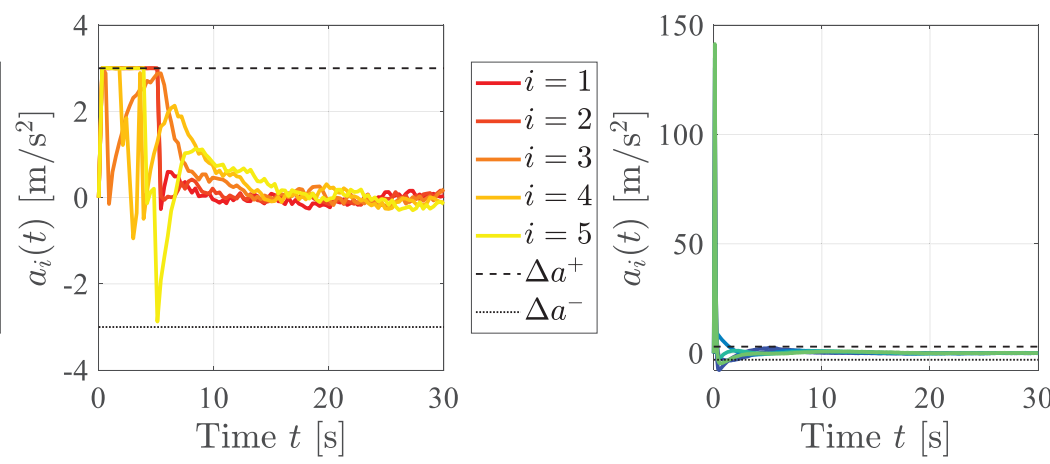

(b) Disturbance Type II
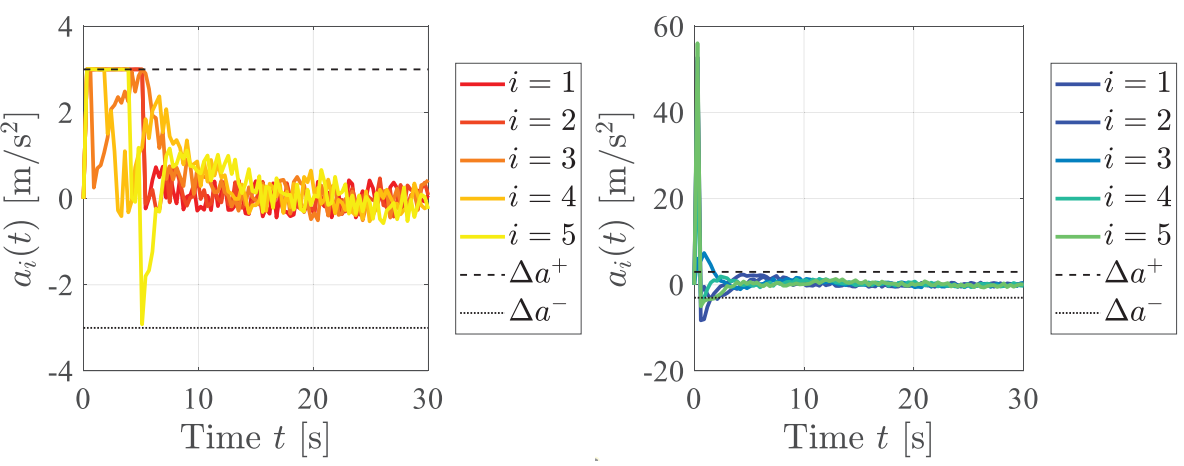

(c) Disturbance Type III
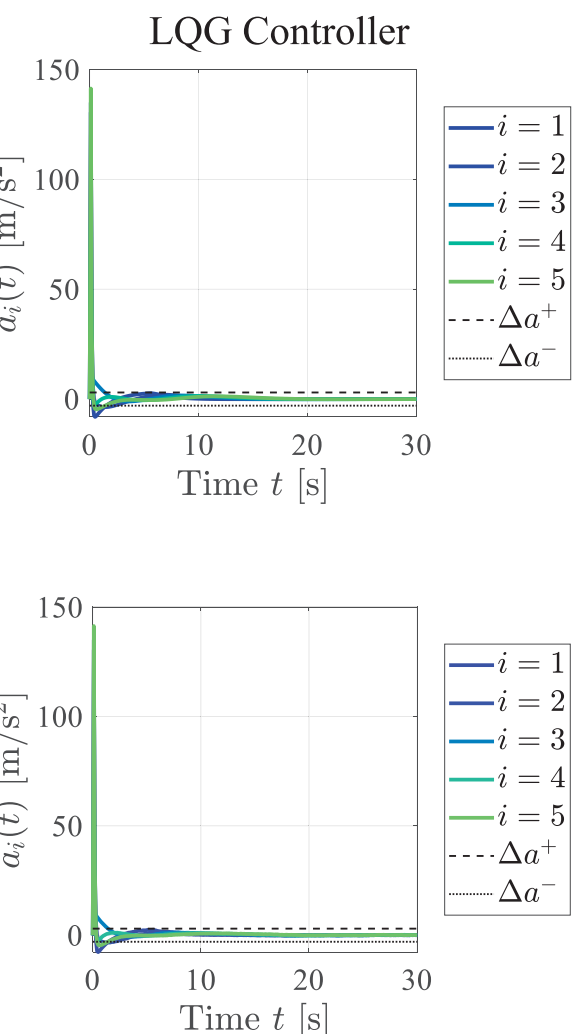

Time $t[\mathrm{~s}]$

Fig. 9. The longitudinal acceleration profiles of the vehicles with different platoon controllers under different disturbances.

additional simulation experiments, in which the platoon is set to a larger size, i.e., increasing the vehicle number to $N=10$. Moreover, the positions and velocities of the vehicles are initialized by random perturbations of the equilibrium state. That is, the initial space headway and initial velocity of each vehicle in the platoon are non-zero and different from each other's. The reference velocity provided by the virtual leading vehicle is specified to be time-varying, which is shown by the curve of $v_{\text {desired }}$ in Figure 11. In particular, the virtual leading vehicle is considered to experience not only a normal driving stage but also sudden acceleration and sudden deceleration stages. In this way, both the emergent acceleration and braking situations are simulated and experienced by the platooning vehicles in order to sufficiently validate the proposed control method. Besides, we take into account the impacts of exter- nal uncertain disturbances on all the position, velocity and acceleration state equations of each vehicle, and the external disturbances of the vehicles are randomly time-varying and heterogeneous. Simulation results are detailed in Figures 10 to 13 .

The control inputs of the vehicles are depicted in Figure 10. It is seen that the control input of each vehicle suddenly decreases and increases at about $t=40 \mathrm{~s}$ and $t=80 \mathrm{~s}$, respectively. This is because the platooning vehicles need to response to the emergent braking and acceleration of their virtual leading vehicle at these two time instants. Due to the existence of external random disturbances, the control sequences of all the vehicles are perturbed. Nonetheless, the control sequences are strictly bounded within the feasible region $[-5,5]\left(\mathrm{m} / \mathrm{s}^{3}\right)$. From Figure 11, it is observed that, 


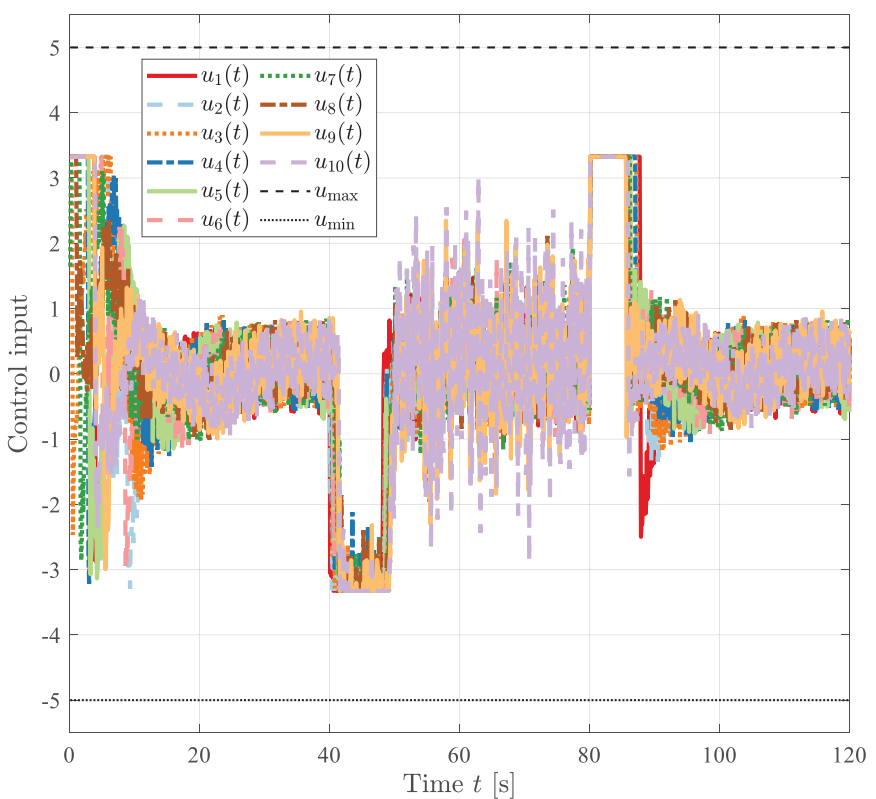

Fig. 10. The control inputs of the vehicles with the designed robust MPC platoon controller based on the causal disturbance feedback.
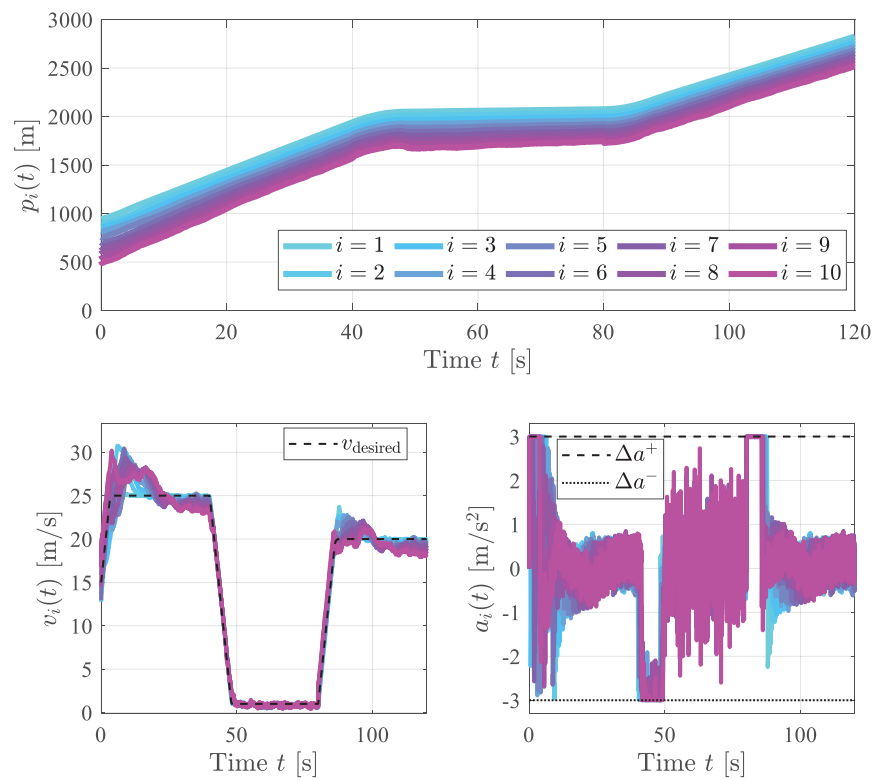

Fig. 11. The longitudinal position, velocity, and acceleration profiles of the vehicles with the designed robust MPC platoon controller based on the causal disturbance feedback.

by utilizing the proposed robust control method, all the vehicles can track the time-varying desired velocity of the virtual leading vehicle. The vehicles can asymptotically converge to around the desired velocity profile even after the virtual leading vehicle performs either emergent braking or emergent acceleration. It is also recognized from 11 that, even in the presence of external uncertain disturbances, the velocity profiles of all the platooning vehicles can be strictly bounded within the feasible region $[0,120 / 3.6](\mathrm{m} / \mathrm{s})$. At the same time, the acceleration profiles of the vehicles are also guaranteed to be ranging within the allowable interval $[-3,3]\left(\mathrm{m} / \mathrm{s}^{2}\right)$.

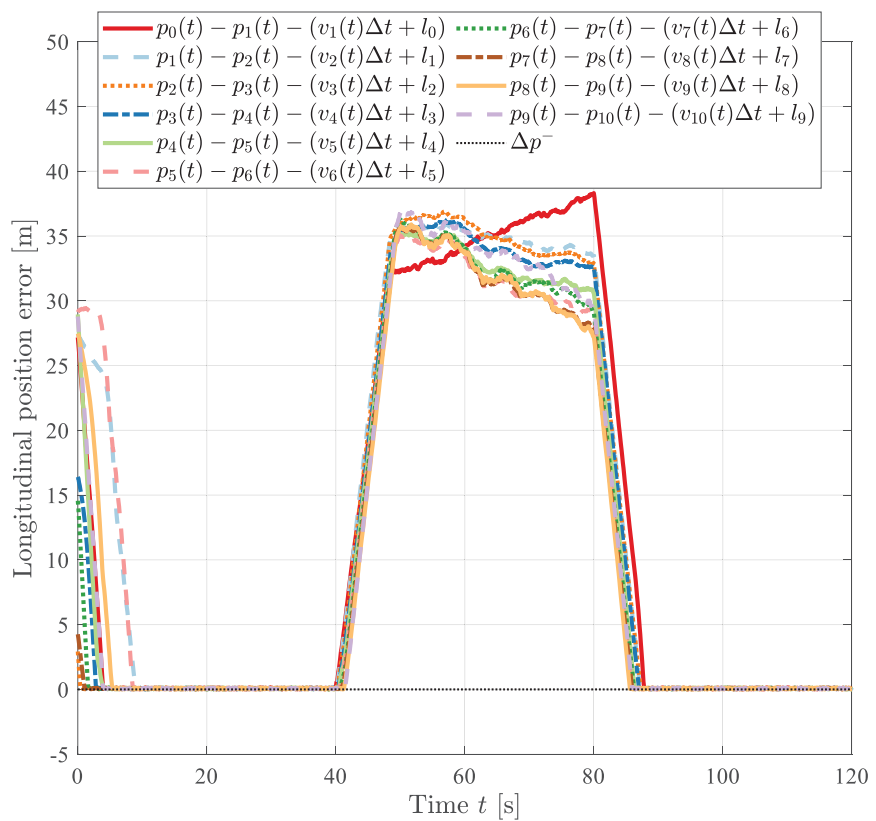

Fig. 12. The longitudinal position errors of the vehicles with the designed robust MPC platoon controller based on the causal disturbance feedback.

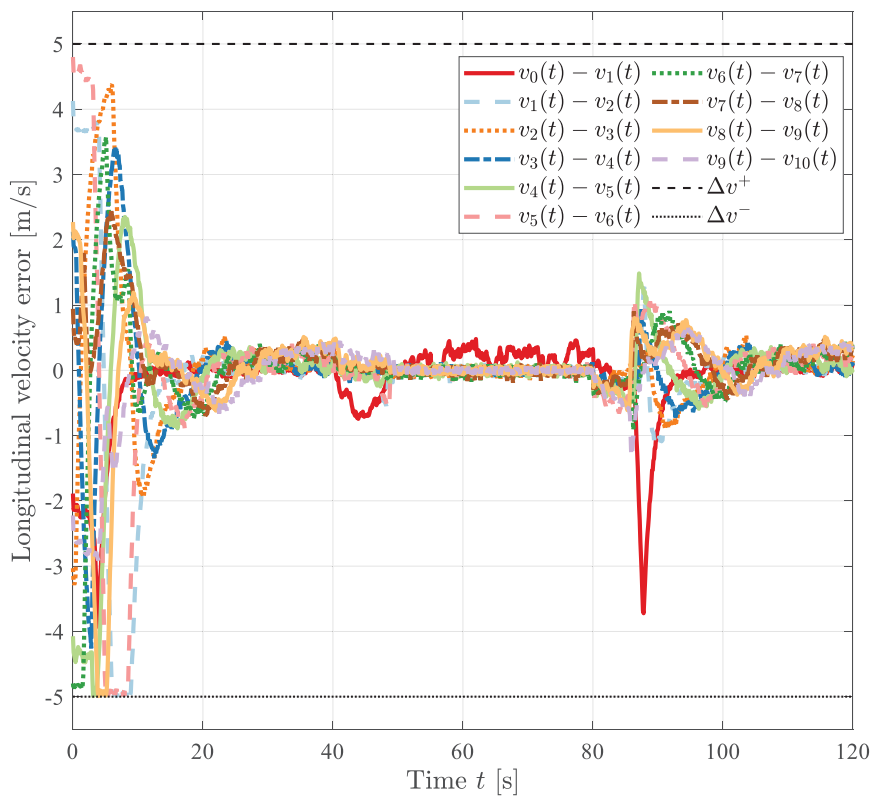

Fig. 13. The longitudinal velocity errors of the vehicles with the designed robust MPC platoon controller based on the causal disturbance feedback.

Figure 12 demonstrates the inter-vehicle spacing errors of the vehicles in the platoon. It is seen that, due to the existence of external random disturbances in all the state equations and the emergent operations of the virtual leading vehicle, the vehicles experience some certain overshoots of inter-vehicle spacing errors within about $[40,90] \mathrm{s}$. In particular, the RMSE of the inter-vehicle spacing errors of the whole platoon from $50 \mathrm{~s}$ to $80 \mathrm{~s}$ is about $33.4267 \mathrm{~m}$ on average. Nonetheless, all the inter-vehicle spaces are maintained above zero, i.e., always ensuring the satisfaction of the position constraint 
$e_{1, i}(k) \geq 0$ for all $i$ and $k$, which implies that the chain collisions can be strictly avoided even under external severe uncertainties. Besides, from Figure 13, we can see that the errors in velocity tracking can asymptotically converge to around zero after the initialization or even after the emergent deceleration and acceleration of the virtual leading vehicle. For example, even during the same interval when the platooning vehicles experience serious overshoots in their inter-vehicle spacing errors, i.e., $[50,80](\mathrm{s})$, the RMSE of the velocity tracking errors of the platoon is only about $0.068812 \mathrm{~m} / \mathrm{s}$ on average. Here, it should be remarked that the errors in both the inter-vehicle spacing and velocity tracking can never be zero since external random disturbances always exist in all the position, velocity and acceleration states of the platoon system. However, it can be seen that, even under both the effects of the external disturbances and the emergent operations, these errors can asymptotically converge and be stabilized near zero. More importantly, the physical constraints are always satisfied in this perturbed platoon system. It can be summarized that the proposed control method can robustly realize vehicle platooning and guarantee the physical constraint satisfaction.

\section{CONCLUSION AND FUtURE WORK}

We have developed a min-max MPC in a closed loop with the causal disturbance feedback for robust vehicle platooning subject to external uncertain disturbances. The min-max MPC model mathematically maintains the convexity of the semidefinite-relaxation transformation by incorporating the disturbance feedback in a causal structure, such that it can be efficiently solved by using current convex optimization techniques. The proposed MPC method has also been proved to be recursively feasible and input-to-state practically stable. Simulations and performance comparisons have been conducted to verify the effectiveness of the proposed method, meanwhile demonstrating its advantage in robustly realizing the platooning objective and satisfying the control constraints against external disturbances. As future work, we will investigate a mixed vehicle platoon where both human-driven and autonomous vehicles coexist. We will also extend the proposed platoon control method to a distributed scenario and explore novel robust modeling and optimization methods, where both state and control can be mixed and optimal control sequences are computed in a distributed manner.

\section{APPENDIX A}

\section{PROOF OF LEMMA 1}

For the $l$-th row of $\boldsymbol{C}$, denoted by $[\boldsymbol{C}]_{l}$, it is obvious that $\max _{|\boldsymbol{x}| \leq 1}\left\{[\boldsymbol{C}]_{l} \boldsymbol{x}\right\}=\max _{|\boldsymbol{x}| \leq 1}\left\{\sum_{\forall l^{\prime}}[\boldsymbol{C}]_{l, l^{\prime}}[\boldsymbol{x}]_{l^{\prime}}\right\}=$ $\sum_{\forall l^{\prime}}[\boldsymbol{C}]_{l, l^{\prime}} \operatorname{sign}\left([\boldsymbol{C}]_{l, l^{\prime}}\right)=\left\|[\boldsymbol{C}]_{l}\right\|_{1}$, where $[\boldsymbol{C}]_{l, l^{\prime}}$ denotes the $\boldsymbol{l}^{\prime}$-th element of $[\boldsymbol{C}]_{l}$ and $[\boldsymbol{x}]_{l^{\prime}}$ is the $\boldsymbol{l}^{\prime}$-th element of $\boldsymbol{x}$. Applying the result for all the rows in the matrix $C$, we can have $\max _{|\boldsymbol{x}| \leq 1}\{\boldsymbol{C} \boldsymbol{x}\} \leq \operatorname{col}\left\{\left\|[\boldsymbol{C}]_{l}\right\|_{1}, \forall l\right\}=|\boldsymbol{C}| \mathbf{1}$.

\section{APPENDIX B}

\section{PROOF OF THEOREM 1}

For the simplicity of notation, let $\boldsymbol{G}_{K}=\boldsymbol{G}_{D}+\boldsymbol{G}_{B} \boldsymbol{K}$. Applying the Schur complement in Lemma 2, we can immediately obtain

$$
\left[\begin{array}{ccc}
\gamma & \boldsymbol{Y}^{\mathrm{T}}+\boldsymbol{D}^{\mathrm{T}} \boldsymbol{G}_{K}^{\mathrm{T}} & \boldsymbol{U}_{K}^{\mathrm{T}}+\boldsymbol{D}^{\mathrm{T}} \boldsymbol{K}^{\mathrm{T}} \\
\boldsymbol{Y}+\boldsymbol{G}_{K} \boldsymbol{D} & \boldsymbol{W}_{X}^{-1} & \mathbf{0}_{3 H N \times H N} \\
\boldsymbol{U}_{K}+\boldsymbol{K} \boldsymbol{D} & \mathbf{0}_{H N \times 3 H N} & \boldsymbol{W}_{U}^{-1}
\end{array}\right] \succeq 0,
$$

for all $\boldsymbol{D} \in \mathbb{D}$. Now, we separate the deterministic and the uncertain terms from (51) above to derive

$$
\begin{aligned}
& {\left[\begin{array}{ccc}
\gamma & \boldsymbol{Y}^{\mathrm{T}} & \boldsymbol{U}_{K}^{\mathrm{T}} \\
\boldsymbol{Y} & \boldsymbol{W}_{X}^{-1} & \mathbf{0}_{3 H N \times H N} \\
\boldsymbol{U}_{K} & \mathbf{0}_{H N \times 3 H N} & \boldsymbol{W}_{U}^{-1}
\end{array}\right] } {\left[\begin{array}{c}
\mathbf{0}_{1 \times 3 H N} \\
\boldsymbol{G}_{K} \\
\boldsymbol{K}
\end{array}\right] \boldsymbol{D}\left[\begin{array}{lll}
1 & \mathbf{0}_{1 \times 3 H N} & \mathbf{0}_{1 \times H N}
\end{array}\right] } \\
&+\left[\begin{array}{c}
1 \\
\mathbf{0}_{3 H N \times 1} \\
\mathbf{0}_{H N \times 1}
\end{array}\right] \boldsymbol{D}^{\mathrm{T}}\left[\begin{array}{lll}
\mathbf{0}_{3 H N \times 1} & \boldsymbol{G}_{K}^{\mathrm{T}} & \boldsymbol{K}^{\mathrm{T}}
\end{array}\right] \succeq 0,
\end{aligned}
$$

for all $\boldsymbol{D} \in \mathbb{D}$. Substituting (44) into (52) can result in (45). At this point, the theorem is proven.

\section{APPENDIX C \\ PROOF OF THEOREM 2}

To prove the theorem here, we first decompose the diagonal matrix $S(\lambda)$ into a weighed summation of a series of submatrices $\boldsymbol{S}_{l} \boldsymbol{S}_{l}^{\mathrm{T}}, l=1,2, \ldots, 3 H N$, i.e.,

$$
\boldsymbol{S}(\lambda)=\sum_{l=1}^{3 H N} \lambda_{l} \boldsymbol{S}_{l} \boldsymbol{S}_{l}^{\mathrm{T}},
$$

where $S_{l}$ is a $3 H N \times 1$ unit column vector whose $l$-th element is equal to 1 while the others are zeros.

According to the positive semi-definiteness, the uncertain LMI in (45) is equivalent to

$$
\boldsymbol{y}_{1}^{\mathrm{T}}\left(g\left(\boldsymbol{U}_{K}, \gamma\right)+\boldsymbol{L} \boldsymbol{\Delta} \boldsymbol{R}+\boldsymbol{R}^{\mathrm{T}} \boldsymbol{\Delta}^{\mathrm{T}} \boldsymbol{L}^{\mathrm{T}}\right) \boldsymbol{y}_{1} \geq 0
$$

for all $\boldsymbol{\Delta} \in \mathcal{W}$, where $\boldsymbol{y}_{1}$ is any real column vector of a compatible dimension, i.e., $\boldsymbol{y}_{1} \in \mathbb{R}^{(1+3 H N+H N) \times 1}$. In addition, we let

$$
\boldsymbol{y}_{2}=\boldsymbol{\Delta}^{\mathrm{T}} \boldsymbol{L}^{\mathrm{T}} \boldsymbol{y}_{1}
$$

and thus obtain from (54)

$$
\boldsymbol{y}_{1}^{\mathrm{T}} g(\boldsymbol{U}, \gamma) \boldsymbol{y}_{1}+\boldsymbol{y}_{2}^{\mathrm{T}} \boldsymbol{R} \boldsymbol{y}_{1}+\boldsymbol{y}_{1}^{\mathrm{T}} \boldsymbol{R}^{\mathrm{T}} \boldsymbol{y}_{2} \geq 0 .
$$

The inequality (56) can be further reshaped into the following more compact form

$$
\left[\begin{array}{l}
\boldsymbol{y}_{1} \\
\boldsymbol{y}_{2}
\end{array}\right]^{\mathrm{T}}\left[\begin{array}{cc}
g\left(\boldsymbol{U}_{K}, \gamma\right) & \boldsymbol{R}^{\mathrm{T}} \\
\boldsymbol{R} & \mathbf{0}
\end{array}\right]\left[\begin{array}{l}
\boldsymbol{y}_{1} \\
\boldsymbol{y}_{2}
\end{array}\right] \geq 0
$$

From (53), we notice that

$$
\boldsymbol{S}_{l}^{\mathrm{T}} \boldsymbol{\Delta}^{\mathrm{T}}=\boldsymbol{S}_{l}^{\mathrm{T}} \boldsymbol{\Delta}^{\mathrm{T}} \boldsymbol{S}_{l} \boldsymbol{S}_{l}^{\mathrm{T}}, \quad l=1,2, \ldots, 3 H N .
$$

Hence, we can combine (58) with the following

$$
\boldsymbol{S}_{l}^{\mathrm{T}} \boldsymbol{y}_{2}=\boldsymbol{S}_{l}^{\mathrm{T}} \boldsymbol{\Delta}^{\mathrm{T}} \boldsymbol{L}^{\mathrm{T}} \boldsymbol{y}_{1}
$$

to get

$$
\boldsymbol{S}_{l}^{\mathrm{T}} \boldsymbol{y}_{2}=\boldsymbol{S}_{l}^{\mathrm{T}} \boldsymbol{\Delta}^{\mathrm{T}} \boldsymbol{S}_{l} \boldsymbol{S}_{l}^{\mathrm{T}} \boldsymbol{L}^{\mathrm{T}} \boldsymbol{y}_{1}, \quad l=1,2, \ldots, 3 H N .
$$


On the other side, since the uncertainty set $\mathcal{W}$ indicates $\left\|\Delta S_{l}\right\|^{2} \leq 1$ for all $l$, we can get

$$
\boldsymbol{\Delta} \boldsymbol{S}_{l} \boldsymbol{S}_{l}^{\mathrm{T}} \boldsymbol{\Delta}^{\mathrm{T}} \leq \boldsymbol{I}_{3 H N \times 3 H N}, \quad l=1,2, \ldots, 3 H N .
$$

Notice $\boldsymbol{S}_{l}^{\mathrm{T}} \boldsymbol{S}_{l}=1$ for $l=1,2, \ldots, 3 H N$. Multiplying (61) with $\boldsymbol{S}_{l} \boldsymbol{S}_{l}^{\mathrm{T}} \boldsymbol{L}^{\mathrm{T}} \boldsymbol{y}_{1}$ and applying (60) can further obtain

$$
\begin{aligned}
& \left(\boldsymbol{S}_{l} \boldsymbol{S}_{l}^{\mathrm{T}} \boldsymbol{L}^{\mathrm{T}} \boldsymbol{y}_{1}\right)^{\mathrm{T}} \boldsymbol{\Delta} \boldsymbol{S}_{l} \boldsymbol{S}_{l}^{\mathrm{T}} \boldsymbol{\Delta}^{\mathrm{T}}\left(\boldsymbol{S}_{l} \boldsymbol{S}_{l}^{\mathrm{T}} \boldsymbol{L}^{\mathrm{T}} \boldsymbol{y}_{1}\right) \\
& \quad=\left(\boldsymbol{y}_{1}^{\mathrm{T}} \boldsymbol{L} \boldsymbol{S}_{l} \boldsymbol{S}_{l}^{\mathrm{T}} \boldsymbol{\Delta} \boldsymbol{S}_{l}\right)\left(\boldsymbol{S}_{l}^{\mathrm{T}} \boldsymbol{\Delta}^{\mathrm{T}} \boldsymbol{S}_{l} \boldsymbol{S}_{l}^{\mathrm{T}} \boldsymbol{L}^{\mathrm{T}} \boldsymbol{y}_{1}\right) \\
& \quad=\boldsymbol{y}_{2}^{\mathrm{T}} \boldsymbol{S}_{l} \boldsymbol{S}_{l}^{\mathrm{T}} \boldsymbol{y}_{2} \leq\left(\boldsymbol{S}_{l} \boldsymbol{S}_{l}^{\mathrm{T}} \boldsymbol{L}^{\mathrm{T}} \boldsymbol{y}_{1}\right)^{\mathrm{T}}\left(\boldsymbol{S}_{l} \boldsymbol{S}_{l}^{\mathrm{T}} \boldsymbol{L}^{\mathrm{T}} \boldsymbol{y}_{1}\right) \\
& \quad=\boldsymbol{y}_{1}^{\mathrm{T}} \boldsymbol{L} \boldsymbol{S}_{l} \boldsymbol{S}_{l}^{\mathrm{T}} \boldsymbol{S}_{l} \boldsymbol{S}_{l}^{\mathrm{T}} \boldsymbol{L}^{\mathrm{T}} \boldsymbol{y}_{1}=\boldsymbol{y}_{1}^{\mathrm{T}} \boldsymbol{L} \boldsymbol{S}_{l} \boldsymbol{S}_{l}^{\mathrm{T}} \boldsymbol{L}^{\mathrm{T}} \boldsymbol{y}_{1}
\end{aligned}
$$

for $l=1,2, \ldots, 3 H N$. The inequality (62) is equivalent to

$$
\left[\begin{array}{l}
\boldsymbol{y}_{1} \\
\boldsymbol{y}_{2}
\end{array}\right]^{\mathrm{T}}\left[\begin{array}{cc}
\boldsymbol{L} \boldsymbol{S}_{l} \boldsymbol{S}_{l}^{\mathrm{T}} \boldsymbol{L}^{\mathrm{T}} & \mathbf{0} \\
\mathbf{0} & -\boldsymbol{S}_{l} \boldsymbol{S}_{l}^{\mathrm{T}}
\end{array}\right]\left[\begin{array}{l}
\boldsymbol{y}_{1} \\
\boldsymbol{y}_{2}
\end{array}\right] \geq 0
$$

for all $l=1,2, \ldots, 3 H N$.

From both (57) and (63), the theorem is equivalent to state that (63) holds for any compatible $\boldsymbol{y}_{1}$ and $\boldsymbol{y}_{2}$ such that (57) always holds, i.e., $(63) \Rightarrow(57)$. Based on the S-procedure in Lemma 3 , we can obtain a sufficient condition for ensuring the implication as follows

$$
\left[\begin{array}{cc}
g\left(\boldsymbol{U}_{K}, \gamma\right) & \boldsymbol{R}^{\mathrm{T}} \\
\boldsymbol{R} & \mathbf{0}
\end{array}\right] \geq \sum_{l=1}^{3 H N} \lambda_{l}\left[\begin{array}{cc}
\boldsymbol{L} \boldsymbol{S}_{l} \boldsymbol{S}_{l}^{\mathrm{T}} \boldsymbol{L}^{\mathrm{T}} & \mathbf{0} \\
\mathbf{0} & -\boldsymbol{S}_{l} \boldsymbol{S}_{l}^{\mathrm{T}}
\end{array}\right]
$$

which is indeed the result in (46) recalling the definition of $S(\lambda)$ in (53). Hence, the theorem is proven.

\section{APPENDIX D \\ PROOF OF LEMMA 4}

Recall that the external disturbance term $\boldsymbol{D}$ is considered to be bounded within a finite set as $\boldsymbol{D} \in \mathbb{D}$, and the system state $\boldsymbol{X}$ and the control sequence $\boldsymbol{U}$ are also bounded as $\boldsymbol{X} \in \mathbb{X}$ and $\boldsymbol{U} \in \mathbb{U}$, respectively. The boundedness of both the system and its external disturbance indeed guarantees that the system trajectory over the finite prediction horizon will be bounded as well. Based on the boundedness and also as shown in [67], the existence of a RPI set is held due to $\boldsymbol{M}_{A}+\boldsymbol{M}_{B} \boldsymbol{k}_{x}$ being stable. Furthermore, the approximation method proposed in [67] can also be adopted to construct the RPI set $\mathcal{E}(\boldsymbol{V})$.

\section{APPENDIX E \\ ProOF OF THEOREM 3}

To prove the recursive feasibility and the ISpS of the platoon system (10) using the robust min-max MPC control (49), we resort to the definition of ISpS and its theorem developed in [71]. First, according to Lemma 4, we can have a RPI set $\mathcal{E}(\boldsymbol{V})$ for the controlled system and treat $\mathcal{E}(\boldsymbol{V})$ as the terminal constraint set for the system. The invariance of $\mathcal{E}(\boldsymbol{V})$ guarantees the recursive feasibility, i.e., $\boldsymbol{x}(k+i+1 \mid k) \in \mathcal{X}$ since $\boldsymbol{x}(k+i \mid k) \in \mathcal{E}(\boldsymbol{V}) \subseteq \mathcal{X}$ for all $i$. Additionally, since the quadratic function is used for the stage cost $\mathcal{L}(\boldsymbol{x}, \boldsymbol{u})$ and the terminal cost $\Phi(\boldsymbol{x})$, the boundedness of the cost functions can also be met under the state and control bound constraints.
In the following, we only need to show that the controlled system satisfies [71]

$$
\begin{aligned}
& \Phi(\boldsymbol{x}(k+i+1 \mid k))-\Phi(\boldsymbol{x}(k+i \mid k)) \\
& \leq-\mathcal{L}(\boldsymbol{x}(k+i \mid k), \boldsymbol{u}(k+i \mid k)) \\
&+\alpha \boldsymbol{d}^{\mathrm{T}}(k+i \mid k) \boldsymbol{M}_{D}^{\mathrm{T}} \boldsymbol{M}_{D} \boldsymbol{d}(k+i \mid k)+\Delta \Phi
\end{aligned}
$$

for all $\boldsymbol{d}(k+i \mid k) \in \mathcal{D}$, where $\Delta \Phi$ denotes a tolerant nonnegative offset, i.e., $\Delta \Phi \geq 0$, that may exist in reality.

Applying the Schur complement in Lemma 2 to (50) can yield the equivalent form

$$
\begin{gathered}
{\left[\begin{array}{cc}
\boldsymbol{V} & \mathbf{0}_{3 N \times 3 N} \\
\mathbf{0}_{3 N \times 3 N} & \alpha \boldsymbol{M}_{D}^{\mathrm{T}} \boldsymbol{M}_{D}
\end{array}\right]-\left[\begin{array}{c}
\boldsymbol{M}_{K}^{\mathrm{T}} \\
\boldsymbol{M}_{D}^{\mathrm{T}}
\end{array}\right] \boldsymbol{V}\left[\begin{array}{ll}
\boldsymbol{M}_{K} & \boldsymbol{M}_{D}
\end{array}\right]} \\
-\left[\begin{array}{c}
\boldsymbol{P}_{x}^{\mathrm{T}} \\
\mathbf{0}_{3 N \times 3 N}
\end{array}\right] \boldsymbol{I}_{3 N \times 3 N}\left[\begin{array}{ll}
\boldsymbol{P}_{x} & \mathbf{0}_{3 N \times 3 N}
\end{array}\right] \\
-\left[\begin{array}{c}
\boldsymbol{k}_{x}^{\mathrm{T}} \boldsymbol{P}_{u}^{\mathrm{T}} \\
\mathbf{0}_{3 N \times N}
\end{array}\right] \boldsymbol{I}_{N \times N}\left[\begin{array}{ll}
\boldsymbol{P}_{u} \boldsymbol{k}_{x} & \left.\mathbf{0}_{N \times 3 N}\right] \succeq 0 .
\end{array}\right.
\end{gathered}
$$

From (66), we can see that for any $\boldsymbol{x} \in \mathcal{X}$ and $\boldsymbol{d} \in \mathcal{D}$

$$
\begin{aligned}
& {\left[\begin{array}{ll}
\boldsymbol{x}^{\mathrm{T}} & \boldsymbol{d}^{\mathrm{T}}
\end{array}\right]\left[\begin{array}{cc}
\boldsymbol{V}-\boldsymbol{w}_{x}-\boldsymbol{k}_{x}^{\mathrm{T}} \boldsymbol{w}_{u} \boldsymbol{k}_{x} & \mathbf{0}_{3 N \times 3 N} \\
\mathbf{0}_{3 N \times 3 N} & \alpha \boldsymbol{M}_{D}^{\mathrm{T}} \boldsymbol{M}_{D}
\end{array}\right]\left[\begin{array}{l}
\boldsymbol{x} \\
\boldsymbol{d}
\end{array}\right]} \\
& -\left[\begin{array}{ll}
\boldsymbol{x}^{\mathrm{T}} & \boldsymbol{d}^{\mathrm{T}}
\end{array}\right]\left[\begin{array}{lll}
\boldsymbol{M}_{K}^{\mathrm{T}} \boldsymbol{V} \boldsymbol{M}_{K} & \boldsymbol{M}_{K}^{\mathrm{T}} \boldsymbol{V} \boldsymbol{M}_{D} \\
\boldsymbol{M}_{D}^{\mathrm{T}} \boldsymbol{V} \boldsymbol{M}_{K} & \boldsymbol{M}_{D}^{\mathrm{T}} \boldsymbol{V} & \boldsymbol{M}_{D}
\end{array}\right]\left[\begin{array}{l}
\boldsymbol{x} \\
\boldsymbol{d}
\end{array}\right] \geq 0 .
\end{aligned}
$$

Substituting $\boldsymbol{x}=\boldsymbol{x}(k+i \mid k)$ and $\boldsymbol{d}=\boldsymbol{d}(k+i \mid k)$ into (67) and recalling $\boldsymbol{x}(k+i+1 \mid k)=\boldsymbol{M}_{K} \boldsymbol{x}(k+i \mid k)+\boldsymbol{M}_{D} \boldsymbol{d}(k+i \mid k)$, we can immediately get

$$
\begin{aligned}
& \Phi(\boldsymbol{x}(k+i+1 \mid k))-\Phi(\boldsymbol{x}(k+i \mid k)) \\
& \leq-\mathcal{L}(\boldsymbol{x}(k+i \mid k), \boldsymbol{u}(k+i \mid k)) \\
& \quad+\alpha \boldsymbol{d}^{\mathrm{T}}(k+i \mid k) \boldsymbol{M}_{D}^{\mathrm{T}} \boldsymbol{M}_{D} \boldsymbol{d}(k+i \mid k)
\end{aligned}
$$

for all $\boldsymbol{d}(k+i \mid k) \in \mathcal{D}$, which can make (65) held. Based on Proposition 4.2 of [71], $\Phi(\boldsymbol{x})$ is indeed a local ISpS Lyapunov function for the closed-loop system. The theorem is proven.

It is remarked that when $\Delta \Phi$ is strictly zero, i.e., $\Delta \Phi=0$, the asymptotical stability of the system can be guaranteed in the input-to-state stability (ISS) sense [71]. However, in practice, the trajectory of the Lyapunov function may not strictly reach zero even under zero disturbance inputs. That is, $\Delta \Phi$ is usually not zero in practice. As stated in [71], ISpS is a weaker property than ISS since it does not require zero disturbance inputs, which makes it more suitable for practical application scenarios. Therefore, we focus on the ISpS framework instead of the ISS to characterize the closed-loop min-max MPC-based system.

\section{REFERENCES}

[1] J. Zhou, D. Tian, Y. Wang, Z. Sheng, X. Duan, and V. C. M. Leung, "Reliability-optimal cooperative communication and computing in connected vehicle systems," IEEE Trans. Mobile Comput., vol. 19, no. 5, pp. 1216-1232, May 2020.

[2] D. Tian, J. Zhou, Z. Sheng, M. Chen, Q. Ni, and V. C. M. Leung, "Self-organized relay selection for cooperative transmission in vehicular ad-hoc networks," IEEE Trans. Veh. Technol., vol. 66, no. 10, pp. 9534-9549, Oct. 2017.

[3] G. Qu, J. Zhou, Z. Sheng, H. Yu, and Y. Ren, "Reliability-guaranteed admission control for mobile computation offloading under Nakagami fading channel," IEEE Wireless Commun. Lett., vol. 10, no. 10, pp. 2195-2199, Oct. 2021. 
[4] D. Jia, K. Lu, J. Wang, X. Zhang, and X. Shen, "A survey on platoonbased vehicular cyber-physical systems," IEEE Commun. Surveys Tuts. vol. 18, no. 1, pp. 263-284, 1st Quart., 2016.

[5] X. Shen, R. Fantacci, and S. Chen, "Internet of vehicles [Scanning the Issue]," Proc. IEEE, vol. 108, no. 2, pp. 242-245, Feb. 2020.

[6] Z. Wang, Y. Bian, S. E. Shladover, G. Wu, S. E. Li, and M. J. Barth, "A survey on cooperative longitudinal motion control of multiple connected and automated vehicles," IEEE Intell. Transp. Syst. Mag., vol. 12, no. 1, pp. 4-24, Dec. 2019.

[7] Y. Liu et al., "Joint communication and computation resource scheduling of a UAV-assisted mobile edge computing system for platooning vehicles," IEEE Trans. Intell. Transp. Syst., early access, Jun. 4, 2021, doi: 10.1109/TITS.2021.3082539.

[8] Z. Niu, X. S. Shen, Q. Zhang, and Y. Tang, "Space-air-ground integrated vehicular network for connected and automated vehicles: Challenges and solutions," Intell. Converged Netw., vol. 1, no. 2, pp. 142-169, Sep. 2020

[9] Y. Bian, S. E. Li, W. Ren, J. Wang, K. Li, and H. X. Liu, "Cooperation of multiple connected vehicles at unsignalized intersections: Distributed observation, optimization, and control," IEEE Trans. Ind. Electron., vol. 67, no. 12, pp. 10744-10754, Dec. 2020.

[10] K. Li, F. Gao, Z. Lin, J. Li, Q. Sun, and S. E. Li, "Robust distributed consensus control of uncertain multiagents interacted by eigenvalue-bounded topologies," IEEE Internet Things J., vol. 7, no. 5, pp. 3790-3798, Feb. 2020.

[11] Y.-C. Lin and H. L. T. Nguyen, "Adaptive neuro-fuzzy predictor-based control for cooperative adaptive cruise control system," IEEE Trans. Intell. Transp. Syst., vol. 21, no. 3, pp. 1054-1063, Mar. 2020.

[12] M. Sybis et al., "Communication aspects of a modified cooperative adaptive cruise control algorithm," IEEE Trans. Intell. Transp. Syst., vol. 20, no. 12, pp. 4513-4523, Dec. 2019.

[13] R. Dang, J. Wang, S. E. Li, and K. Li, "Coordinated adaptive cruise control system with lane-change assistance," IEEE Trans. Intell. Transp. Syst., vol. 16, no. 5, pp. 2373-2383, Oct. 2015.

[14] S. Öncï, J. Ploeg, N. van de Wouw, and H. Nijmeijer, "Cooperative adaptive cruise control: Network-aware analysis of string stability," IEEE Trans. Intell. Transp. Syst., vol. 15, no. 4, pp. 1527-1537, Aug. 2014.

[15] F. Navas, V. Milanes, C. Flores, and F. Nashashibi, "Multi-model adaptive control for CACC applications," IEEE Trans. Intell. Transp. Syst., vol. 22, no. 2, pp. 1206-1216, Feb. 2021.

[16] B. Gao, K. Cai, T. Qu, Y. Hu, and H. Chen, "Personalized adaptive cruise control based on online driving style recognition technology and model predictive control," IEEE Trans. Veh. Technol., vol. 69, no. 11, pp. 12482-12496, Nov. 2020.

[17] S. Cheng, L. Li, M.-M. Mei, Y.-L. Nie, and L. Zhao, "Multiple-objective adaptive cruise control system integrated with DYC," IEEE Trans. Veh. Technol., vol. 68, no. 5, pp. 4550-4559, May 2019.

[18] Y. Zhu, D. Zhao, and H. He, "Synthesis of cooperative adaptive cruise control with feedforward strategies," IEEE Trans. Veh. Technol., vol. 69, no. 4, pp. 3615-3627, Apr. 2020.

[19] F. Ma et al., "Distributed control of cooperative vehicular platoon with nonideal communication condition," IEEE Trans. Veh. Technol., vol. 69, no. 8, pp. 8207-8220, Aug. 2020.

[20] H. Xing, J. Ploeg, and H. Nijmeijer, "Compensation of communication delays in a cooperative ACC system," IEEE Trans. Veh. Technol., vol. 69 no. 2, pp. 1177-1189, Feb. 2020.

[21] H. Xing, J. Ploeg, and H. Nijmeijer, "Smith predictor compensating for vehicle actuator delays in cooperative ACC systems," IEEE Trans. Veh. Technol., vol. 68, no. 2, pp. 1106-1115, Feb. 2019.

[22] Y. Li, C. Tang, S. Peeta, and Y. Wang, "Nonlinear consensus-based connected vehicle platoon control incorporating car-following interactions and heterogeneous time delays," IEEE Trans. Intell. Transp. Syst., vol. 20, no. 6, pp. 2209-2219, Jun. 2019.

[23] M. di Bernardo, A. Salvi, and S. Santini, "Distributed consensus strategy for platooning of vehicles in the presence of time-varying heterogeneous communication delays," IEEE Trans. Intell. Transp. Syst., vol. 16, no. 1, pp. 102-112, Feb. 2015.

[24] S. Santini, A. Salvi, A. S. Valente, A. Pescapé, M. Segata, and R. L. Cigno, "A consensus-based approach for platooning with intervehicular communications and its validation in realistic scenarios," IEEE Trans. Veh. Technol., vol. 66, no. 3, pp. 1985-1999, Mar. 2017.

[25] W. Levine and M. Athans, "On the optimal error regulation of a string of moving vehicles," IEEE Trans. Autom. Control, vol. 11, no. 3, pp. 355-361, Jul. 1966.
[26] K.-C. Chu, "Decentralized control of high-speed vehicular strings," Transp. Sci., vol. 8, no. 4, pp. 361-384, 1974. [Online]. Available: http://www.jstor.org/stable/25767760

[27] S. E. Shladover et al., "Automated vehicle control developments in the PATH program," IEEE Trans. Veh. Technol., vol. 40, no. 1, pp. 114-130, Feb. 1991.

[28] Y. Zhou and S. Ahn, "Robust local and string stability for a decentralized car following control strategy for connected automated vehicles," Transp. Res. B, Methodol., vol. 125, pp. 175-196, Jul. 2019. [Online]. Available: https://www.sciencedirect.com/science/article/pii/S0191261518306234

[29] X. Xu, J. W. Grizzle, P. Tabuada, and A. D. Ames, "Correctness guarantees for the composition of lane keeping and adaptive cruise control," IEEE Trans. Autom. Sci. Eng., vol. 15, no. 3, pp. 1216-1229, Jul. 2018

[30] I. Karafyllis, D. Theodosis, and M. Papageorgiou, "Nonlinear adaptive cruise control of vehicular platoons," Int. J. Control, pp. 1-23, 2021, doi: 10.1080/00207179.2021.1982015.

[31] C. K. Verginis, C. P. Bechlioulis, D. V. Dimarogonas, and K. J. Kyriakopoulos, "Robust distributed control protocols for large vehicular platoons with prescribed transient and steady-state performance," IEEE Trans. Control Syst. Technol., vol. 26, no. 1, pp. 299-304, Jan. 2018.

[32] B. Besselink and K. H. Johansson, "String stability and a delay-based spacing policy for vehicle platoons subject to disturbances," IEEE Trans. Autom. Control, vol. 62, no. 9, pp. 4376-4391, Sep. 2017.

[33] Y. Wu, S. E. Li, J. Cortes, and K. Poolla, "Distributed sliding mode control for nonlinear heterogeneous platoon systems with positive definite topologies," IEEE Trans. Control Syst. Technol., vol. 28, no. 4, pp. 1272-1283, Jul. 2020.

[34] Y.-J. Pan, "Decentralized robust control approach for coordinated maneuvering of vehicles in platoons," IEEE Trans. Intell. Transp. Syst., vol. 10, no. 2, pp. 346-354, Jun. 2009.

[35] F. Gao, X. Hu, S. E. Li, K. Li, and Q. Sun, "Distributed adaptive sliding mode control of vehicular platoon with uncertain interaction topology," IEEE Trans. Ind. Electron., vol. 65, no. 8, pp. 6352-6361, Aug. 2018.

[36] J. Wang, X. Luo, J. Yan, and X. Guan, "Distributed integrated sliding mode control for vehicle platoons based on disturbance observer and multi power reaching law," IEEE Trans. Intell. Transp. Syst., early access, Nov. 17, 2020, doi: 10.1109/TITS.2020.3035764.

[37] J. Wang, X. Luo, L. Wang, Z. Zuo, and X. Guan, "Integral sliding mode control using a disturbance observer for vehicle platoons," IEEE Trans. Ind. Electron., vol. 67, no. 8, pp. 6639-6648, Aug. 2020.

[38] X. Guo, J. Wang, F. Liao, R. Teo, and H. Teo, "Distributed adaptive sliding mode control strategy for vehicle-following systems with nonlinear acceleration uncertainties," IEEE Trans. Veh. Technol., vol. 66, no. 2, pp. 981-991, Feb. 2017.

[39] H. Kwakernaak, "Robust control and $\mathrm{H}_{\infty}$-optimization-Tutorial paper," Automatica, vol. 29, no. 2, pp. 255-273, 1993. [Online]. Available: https://www.sciencedirect.com/science/article/pii/000510989390122A

[40] B. M. Chen, Continuous-Time $H_{\infty}$ Almost Disturbance Decoupling. London, U.K.: Springer, 2000, pp. 177-214. [Online]. Available: https://doi.org/10.1007/978-1-4471-3653-8_8

[41] S. E. Li, F. Gao, D. Cao, and K. Li, "Multiple-model switching control of vehicle longitudinal dynamics for platoon-level automation," IEEE Trans. Veh. Technol., vol. 65, no. 6, pp. 4480-4492, Jun. 2016.

[42] S. E. Li, F. Gao, K. Li, L.-Y. Wang, K. You, and D. Cao, "Robust longitudinal control of multi-vehicle systems-A distributed H-infinity method," IEEE Trans. Intell. Transp. Syst., vol. 19, no. 9, pp. 2779-2788, Sep. 2018.

[43] Y. Zhou, S. Ahn, M. Wang, and S. Hoogendoorn, "Stabilizing mixed vehicular platoons with connected automated vehicles: An H-infinity approach," Transp. Res. B, Methodol., vol. 132, pp. 152-170, Feb. 2020. [Online]. Available: https://www.sciencedirect.com/science/article/pii/S0191261518311482

[44] Y. Zheng, S. E. Li, K. Li, and W. Ren, "Platooning of connected vehicles with undirected topologies: Robustness analysis and distributed H-infinity controller synthesis," IEEE Trans. Intell. Transp. Syst., vol. 19, no. 5, pp. 1353-1364, May 2018.

[45] D. Q. Mayne, J. B. Rawlings, C. V. Rao, and P. O. M. Scokaert, "Constrained model predictive control: Stability and optimality," Automatica, vol. 36, no. 6, pp. 789-814, 2000. [Online]. Available: https://www.sciencedirect.com/science/article/pii/S0005109899002149

[46] Y. Zheng, S. E. Li, K. Li, F. Borrelli, and J. K. Hedrick, "Distributed model predictive control for heterogeneous vehicle platoons under unidirectional topologies," IEEE Trans. Control Syst. Technol., vol. 25, no. 3, pp. 899-910, May 2017. 
[47] S. Gong, J. Shen, and L. Du, "Constrained optimization and distributed computation based car following control of a connected and autonomous vehicle platoon," Transp. Res. B, Methodol., vol. 94, pp. 314-334, Dec. 2016. [Online]. Available: https://www.sciencedirect.com/science/article/pii/S0191261516303836

[48] M. Wang, W. Daamen, S. P. Hoogendoorn, and B. van Arem, "Rolling horizon control framework for driver assistance systems. Part I: Mathematical formulation and non-cooperative systems," Transp. Res. C, Emerg. Technol., vol. 40, pp. 271-289, Mar. 2014. [Online]. Available: https://www.sciencedirect.com/science/article/pii/S0968090X13002593

[49] M. Wang, W. Daamen, S. P. Hoogendoorn, and B. van Arem, "Rolling horizon control framework for driver assistance systems. Part II: Cooperative sensing and cooperative control," Transp. Res. C, Emerg. Technol., vol. 40, pp. 290-311, Mar. 2014. [Online]. Available: https://www.sciencedirect.com/science/article/pii/S0968090X13002611

[50] M. Lorenzen, M. Cannon, and F. Allgöwer, "Robust MPC with recursive model update," Automatica, vol. 103, pp. 461-471, May 2019. [Online]. Available: https://www.sciencedirect. com/science/article/pii/S0005109819300731

[51] F. D. Brunner, M. Heemels, and F. Allgöwer, "Robust selftriggered MPC for constrained linear systems: A tube-based approach," Automatica, vol. 72, pp. 73-83, Oct. 2016. [Online]. Available: https://www.sciencedirect.com/science/article/pii/S0005109816301881

[52] S. Feng, Z. Song, Z. Li, Y. Zhang, and L. Li, "Robust platoon control in mixed traffic flow based on tube model predictive control," IEEE Trans. Intell. Vehicles, vol. 6, no. 4, pp. 711-722, Dec. 2021.

[53] Y. Zhou, S. Ahn, M. Chitturi, and D. A. Noyce, "Rolling horizon stochastic optimal control strategy for ACC and CACC under uncertainty," Transp. Res. C, Emerg. Technol., vol. 83, pp. 61-76, Oct. 2017. [Online]. Available: https://www.sciencedirect. com/science/article/pii/S0968090X17301997

[54] D. Moser, R. Schmied, H. Waschl, and L. del Re, "Flexible spacing adaptive cruise control using stochastic model predictive control," IEEE Trans. Control Syst. Technol., vol. 26, no. 1, pp. 114-127, Jan. 2018.

[55] J. Lofberg, "Approximations of closed-loop minimax MPC," in Proc. 42nd IEEE Int. Conf. Decis. Control, vol. 2, Dec. 2003, pp. 1438-1442.

[56] J. Löfberg, "Minimax approaches to robust model predictive control," Ph.D. dissertation, Dept. Elect. Eng., Linköping Univ., Linköping, Sweden, 2003.

[57] P. O. M. Scokaert and D. Q. Mayne, "Min-max feedback model predictive control for constrained linear systems," IEEE Trans. Autom. Control, vol. 43, no. 8, pp. 1136-1142, Aug. 1998.

[58] S. Boyd, L. El Ghaoui, E. Feron, and V. Balakrishnan, Linear Matrix Inequalities in System and Control Theory, vol. 15. Philadelphia, PA, USA: SIAM, 1994

[59] L. Dai, F. Yang, Z. Qiang, and Y. Xia, "Robust self-triggered MPC with fast convergence for constrained linear systems," J. Franklin Inst., vol. 356, no. 3, pp. 1446-1467, Feb. 2019. [Online]. Available: https://www.sciencedirect.com/science/article/pii/S0016003219300018

[60] C. Liu, H. Li, J. Gao, and D. Xu, "Robust self-triggered minmax model predictive control for discrete-time nonlinear systems," Automatica, vol. 89, no. 89, pp. 333-339, 2018. [Online]. Available: https://www.sciencedirect.com/science/article/pii/S0005109817306210

[61] N. Chen, M. Wang, T. Alkim, and B. van Arem, "A robust longitudinal control strategy of platoons under model uncertainties and time delays," J. Adv. Transp., vol. 2018, Apr. 2018, Art. no. 9852721, doi: $10.1155 / 2018 / 9852721$

[62] J. Lan and D. Zhao, "Min-max model predictive vehicle platooning with communication delay," IEEE Trans. Veh. Technol., vol. 69, no. 11, pp. 12570-12584, Nov. 2020.

[63] K. Yi and Y. D. Kwon, "Vehicle-to-vehicle distance and speed control using an electronic-vacuum booster," JSAE Rev. vol. 22, no. 4, pp. 403-412, Oct. 2001. [Online]. Available: https://www.sciencedirect.com/science/article/pii/S0389430401001230

[64] M. Wang, S. P. Hoogendoorn, W. Daamen, B. van Arem, B. Shyrokau, and R. Happee, "Delay-compensating strategy to enhance string stability of adaptive cruise controlled vehicles," Transportmetrica B, Trans. Dyn., vol. 6, no. 3, pp. 211-229, 2016. [Online]. Available: https://doi.org/10.1080/21680566.2016.1266973

[65] F. Zhang, Block Matrix Techniques. Boston, MA, USA: Springer, 2005, pp. 83-110. [Online]. Available: https://doi.org/10.1007/0-387-2427324

[66] S. Boyd and L. Vandenberghe, Convex Optimization. Cambridge, U.K.: Cambridge Univ. Press, Mar. 2004. [Online]. Available: http://www. amazon.com/exec/obidos/redirect?tag=citeulike20\&path=ASIN/0521833787
[67] S. V. Rakovic, E. C. Kerrigan, K. I. Kouramas, and D. Q. Mayne, "Invariant approximations of the minimal robust positively Invariant set," IEEE Trans. Autom. Control, vol. 50, no. 3, pp. 406-410, Mar. 2005.

[68] J. Lofberg, "YALMIP: A toolbox for modeling and optimization in MATLAB," in Proc. IEEE Int. Conf. Robot. Autom., Taipei, Taiwan, Sep. 2004, pp. 284-289.

[69] J. Löfberg, "Automatic robust convex programming," Optim. Methods Softw., vol. 27, no. 1, pp. 115-129, Sep. 2012.

[70] MOSEK. (2021). The MOSEK Optimization Tools Version 3.2 (Revision 8). [Online]. Available: http://www.mosek.com

[71] M. Lazar, D. Muñoz de la Peña, W. Heemels, and T. Alamo, "On input-to-state stability of min-max nonlinear model predictive control," Syst. Control Lett., vol. 57, no. 1, pp. 39-48, 2008. [Online]. Available: https://www.sciencedirect.com/science/article/pii/S0167691107000837

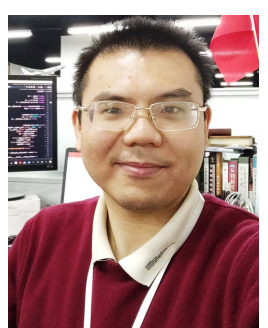

Jianshan Zhou received the B.Sc., M.Sc., and $\mathrm{Ph} . \mathrm{D}$. degrees in traffic information engineering and control from Beihang University, Beijing, China, in 2013, 2016, and 2020, respectively. From 2017 to 2018, he was a Visiting Research Fellow with the School of Informatics and Engineering, University of Sussex, Brighton, U.K. He is currently a Post-Doctoral Research Fellow supported by the Zhuoyue Program of Beihang University and the National Postdoctoral Program for Innovative Talents. He is the author or coauthor of more than 20 international scientific publications. His research interests include the modeling and optimization of vehicular communication networks and air-ground cooperative networks, the analysis and control of connected autonomous vehicles, and intelligent transportation systems. He was the Technical Program Session Chair with the IEEE EDGE 2020, the TPC Member with the IEEE VTC2021-Fall Track, and the Youth Editorial Board Member of the Unmanned Systems Technology. He was a recipient of the First Prize in the Science and Technology Award from the China Intelligent Transportation Systems Association in 2017, the First Prize in the Innovation and Development Award from the China Association of Productivity Promotion Centers in 2020, the National Scholarships in 2017 and 2019, the Outstanding Top-Ten Ph.D. Candidate Prize from Beihang University in 2018, the Outstanding China-SAE Doctoral Dissertation Award in 2020, and the Excellent Doctoral Dissertation Award from Beihang University in 2021

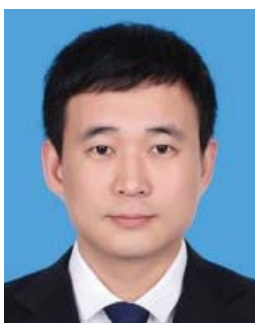

Daxin Tian (Senior Member, IEEE) received the Ph.D. degree in computer application technology from Jilin University, Changchun, China, in 2007. He is currently a University Professor with the School of Transportation Science and Engineering, Beihang University, Beijing, China. His research interests include intelligent transportation systems, autonomous connected vehicles, swarm intelligence, and mobile computing. He was awarded the Changjiang Scholars Program (Young Scholar) of Ministry of Education of China in 2017, the National Science Fund for Distinguished Young Scholars in 2018, and the Distinguished Young Investigator of China Frontiers of Engineering in 2018. 


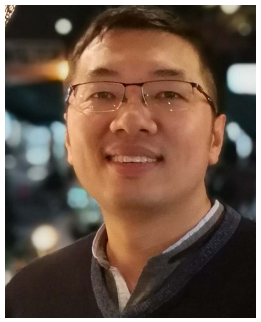

Zhengguo Sheng (Senior Member, IEEE) received the B.Sc. degree from the University of Electronic Science and Technology of China, Chengdu, China, in 2006, and the M.S. and Ph.D. degrees from Imperial College London, London, U.K., in 2007 and 2011, respectively. He is currently a Senior Lecturer with the University of Sussex, Brighton, U.K. Previously, he was with UBC, Vancouver, BC, Canada, as a Research Associate, and with Orange Labs, Santa Monica, CA, USA, as a Senior Researcher. He has more than 100 publications. His research interests include the IoT, vehicular communications, and cloud/edge computing.

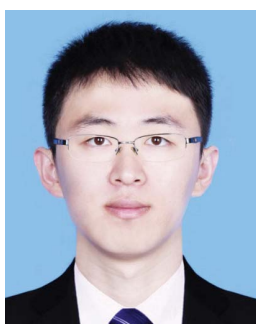

Xuting Duan received the Ph.D. degree in traffic information engineering and control from Beihang University, Beijing, China, in 2017. He is currently an Assistant Professor with the School of Transportation Science and Engineering, Beihang University. His current research interest includes vehicular ad hoc networks.

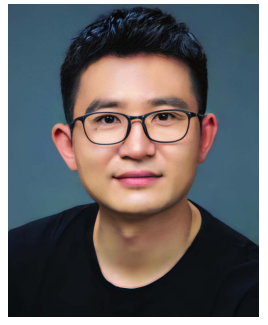

Dongpu Cao (Member, IEEE) received the $\mathrm{Ph} . \mathrm{D}$. degree from Concordia University, Canada, in 2008.

$\mathrm{He}$ is currently an Associate Professor and the Director of the Waterloo Cognitive Autonomous Driving (CogDrive) Laboratory, University of Waterloo, Canada. He is the Canada Research Chair in driver cognition and automated driving. He has contributed more than 200 papers and three books. His current research interests include driver cognition, automated driving, and cognitive autonomous driving. He received the SAE Arch T. Colwell Merit Award in 2012, IEEE VTS 2020 Best Vehicular Electronics Paper Award, and the three Best Paper Awards from the ASME and IEEE conferences. He serves as the Deputy Editor-in-Chief for IET Intelligent Transport Systems journal and an Associate Editor for IEEE TRANSACTIONS ON VEHICULAR TECHNOLOGY, IEEE TRANSACTIONS ON INTELLIGENT TRANSPORTATION SYSTEMS, IEEE/ASME TRANSACTIONS ON MECHATRONICS, IEEE TRANSACTIONS ON INDUSTRIAL ELECTRONICS, IEEE/CAA JOURNAL of Automatica Sinica, IEEE Transactions on Computational Social SYSTEMS, and Journal of Dynamic Systems, Measurement and Control (ASME). He was a Guest Editor of Vehicle System Dynamics, IEEE TRANSACTIONS ON SYSTEMS, MAN, AND CYBERNETICS-PART A: Systems, and IEEE INTERNET OF THINGS JOURNAL. He serves on the SAE Vehicle Dynamics Standards Committee and acts as the Co-Chair for IEEE ITSS Technical Committee on Cooperative Driving. He is an IEEE VTS Distinguished Lecturer.

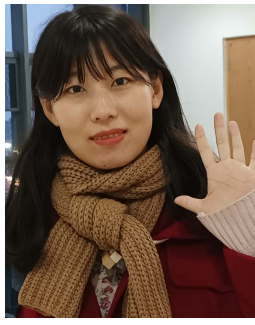

Guixian Qu received the B.Sc. degree in transportation engineering from the Shandong University of Technology, Shandong, China, in 2012, and the M.Sc. and Ph.D. degrees from the Beijing University of Technology, Beijing, China, in 2014 and 2019, respectively. She was a Post-Doctoral Research Fellow with Beihang University. She is currently a Research Fellow with the Research Institute of AeroEngine, Beihang University. Her research interests include intelligent transportation systems, dynamics modeling and control, and collaborative design of aero-engine systems.

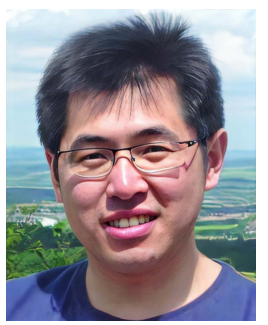

Dezong Zhao (Senior Member, IEEE) received the B.S. and M.S. degrees in control science and engineering from Shandong University, Jinan, China, in 2003 and 2006, respectively, and the Ph.D. degree in control science and engineering from Tsinghua University, Beijing, China, in 2010. Since 2017, he has been a Lecturer with the Department of Aeronautical and Automotive Engineering, Loughborough University, Loughborough, U.K. Since 2020, he has been a Senior Lecturer with the James Watt School of Engineering, University of Glasgow. His research interests include connected and autonomous vehicles, machine learning, and control engineering. He has been an EPSRC Innovation Fellow since 2018 and a Royal Society-Newton Advanced Fellow since 2020. He is a fellow of the Higher Education Academy and the Winner of the Excellence 100 Campaign at Loughborough University.

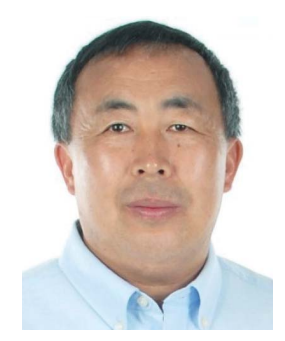

Xuemin (Sherman) Shen (Fellow, IEEE) received the $\mathrm{Ph} . \mathrm{D}$. degree in electrical engineering from Rutgers University, New Brunswick, NJ, USA, in 1990.

$\mathrm{He}$ is currently a University Professor with the Department of Electrical and Computer Engineering, University of Waterloo, Canada. His research interests include network resource management, wireless network security, the Internet of Things, 5G and beyond, and vehicular ad hoc and sensor networks. $\mathrm{He}$ is a registered Professional Engineer of Ontario, Canada, an Engineering Institute of Canada Fellow, a Canadian Academy of Engineering Fellow, a Royal Society of Canada Fellow, a Chinese Academy of Engineering Foreign Member, and a Distinguished Lecturer of the IEEE Vehicular Technology Society and Communications Society. He received the R.A. Fessenden Award from IEEE, Canada, in 2019, the Award of Merit from the Federation of Chinese Canadian Professionals (Ontario) in 2019, the James Evans Avant Garde Award from the IEEE Vehicular Technology Society in 2018, the Joseph LoCicero Award in 2015 and Education Award in 2017 from the IEEE Communications Society, and the Technical Recognition Award from Wireless Communications Technical Committee (2019) and AHSN Technical Committee (2013). He has also received the Excellent Graduate Supervision Award from the University of Waterloo in 2006 and the Premier's Research Excellence Award (PREA) from the Province of Ontario, Canada, in 2003. He served as the Technical Program Committee Chair/Co-Chair for IEEE Globecom'16, IEEE Infocom'14, IEEE VTC'10 Fall, IEEE Globecom'07, and the Chair for the IEEE Communications Society Technical Committee on Wireless Communications. He is the elected IEEE Communications Society Vice President of Technical and Educational Activities, the Vice President of Publications, a Member-at-Large on the Board of Governors, the Chair of the Distinguished Lecturer Selection Committee, and a member of IEEE ComSoc Fellow Selection Committee. $\mathrm{He}$ was/is the Editor-in-Chief of the IEEE INTERNET OF THINGS JOURNAL, IEEE NeTwork, IET Communications, and Peer-to-Peer Networking and Applications. 